\title{
Direct Molecular Simulation of Gradient-Driven Diffusion of Large Molecules using Constant Pressure*
}

\author{
Aidan P. Thompson^ and \\ Grant S. Heffelfinger
}

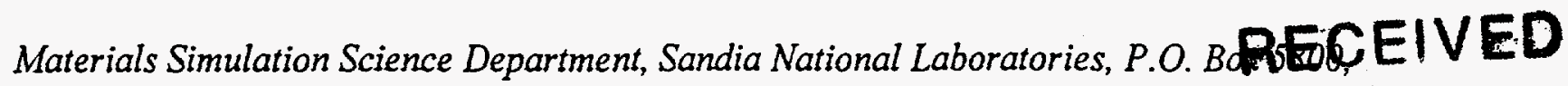
Albuquerque, NM 87185-1111

^author to whom correspondence should be directed

* Sandia is a multiprogram laboratory operated by Sandia Corporation, a Lockheed Martin Company, for the United States Department of Energy under contract number DE-AC04-94AL85000.

\begin{abstract}
Dual control volume grand canonical molecular dynamics (DCV-GCMD) is a boundary-driven non-equilibrium molecular dynamics technique for simulating gradient-driven diffusion in multicomponent systems. Two control volumes are established at opposite ends of the simulation box. Constant temperature and chemical potential of diffusing species are imposed in the control volumes (i.e. constant- $\mu_{1} \ldots \mu_{n-1} \mu_{n} V T$ ). This results in stable chemical potential gradients and steadystate diffusion fluxes in the region between the control volumes. We present results and detailed analysis for a new constant-pressure variant of the DCV-GCMD method in which one of the diffusing species for which a steady-state diffusion flux exists does not have to be inserted or deleted. Constant temperature, pressure and chemical potential of all diffusing species except one are imposed in the control volumes (i.e. constant- $\mu_{1} \ldots \mu_{n-1} N_{n} P T$ ). The constant-pressure method can be applied to situations in which insertion and deletion of large molecules would be prohibitively difficult. As an example, we used the method to simulate diffusion in a binary mixture of spherical particles with a 2:1 size ratio. Steady-state diffusion fluxes of both diffusing species were established. The constant-pressure diffusion coefficients agreed closely with the results of the standard constant-volume calculations.

In addition, we show how the concentration, chemical potential and flux profiles can be used to calculate local binary and Maxwell-Stefan diffusion coefficients. In the case of the 2:1 size ratio mixture, we found that the binary diffusion coefficients were asymmetric and composition dependent, whereas the Maxwell-Stefan diffusion coefficients changed very little with composition and were symmetric. This last result verified that the Gibbs-Duhem relation was satisfied locally, thus validating the assumption of local equilibrium.
\end{abstract}




\section{DISCLAIMER}

This report was prepared as an account of work sponsored by an agency of the United States Government. Neither the United States Government nor any agency thereof, nor any of their employees, make any warranty, express or implied, or assumes any legal liability or responsibility for the accuracy, completeness, or usefulness of any information, apparatus, product, or process disclosed, or represents that its use would not infringe privately owned rights. Reference herein to any specific commercial product, process, or service by trade name, trademark, manufacturer, or otherwise does not necessarily constitute or imply its endorsement, recommendation, or favoring by the United States Government or any agency thereof. The views and opinions of authors expressed herein do not necessarily state or reflect those of the United States Government or any agency thereof. 


\section{DISCLAIMER}

Portions of this document may be illegible in electronic image products. Images are produced from the best available original document. 


\section{Introduction}

In a wide variety of situations of technological and/or scientific interest, the macroscopic behavior of a fluid is influenced by diffusion, which has its origins in the microscopic motions of atoms or molecules comprising the fluid. The term diffusion, in this context, refers to the process whereby a non-equilibrium spatial variation in a species concentration (or more generally chemical potential) causes net motion of that species (relative to the system as a whole) from regions of high concentration to regions of low concentration. The magnitude of the spatial variation can be quantified by some form of concentration or chemical potential gradient, referred to as a driving force. The magnitude of the net relative motion can be quantified by a diffusion flux. While there are many different definitions of these quantities in use in the literature, they are usually defined so that both the driving force and diffusion flux are zero when the system is at equilibrium. Hence, in the absence of unphysical pathologies, the diffusion flux should be directly proportional to the driving force for systems not far removed from equilibrium. The fundamental correctness of this proportionality follows from the principles of non-equilibrium thermodynamics ${ }^{1}$, and it is satisfied in almost all physical situations. The numerical value of the proportionality constant is usually referred to as a diffusion coefficient, although the way in which diffusion coefficients are defined varies widely, particularly in the case of multicomponent diffusion ${ }^{2}$. In any event, the macroscopic phenomenon of diffusion in multicomponent systems can be accurately characterized by a suitable set of diffusion coefficients. A wide variety of experimental techniques for estimating diffusion coefficients have been developed ${ }^{3}$.

The difficulty with experimentally-measured diffusion coefficients is that they fail to provide direct information on the underlying causes of diffusion. Equilibrium molecular dynamics simulations provide a powerful method for examining diffusion at the microscopic level. By sampling the center-of-mass velocity correlation functions (VCF) for every distinct pair of species in the system, complete information about the microscopic translational dynamics, as well as the resultant diffusion coefficients can be obtained. Unfortunately, the VCF's are not self-averaging; their magnitude decays as $1 / N^{4,5}$ This is particularly problematic when large system sizes are needed e.g. in polymeric or coulombic systems. As a consequence, $\mathrm{MD}$ simulations of diffusion frequently sample only the velocity autocorrelation function (VACF). This is a single-particle property which is independent of system size, and so can be sampled very efficiently. The VACF for a species $i$ provides a measure of mobility, which can be used to calculate the tracer diffusion coefficient. This is the ratio of the flux of a very dilute set of tagged particles of species $i$ to a gradient in their concentration, with all other species in the system in their equilibrium states. The tracer diffusion coefficient differs from the full transport diffusion coefficient in that it ignores the effect of collective motion involving molecules of the same species and also molecules of other species. For this reason it is unsuitable for characterizing diffusion of species which are not at very low concentration.

Non-equilibrium molecular dynamics provides an alternative to the difficult-to-sample VCF's, without having to ignore collective motion. The basic principle of $\mathrm{NEMD}^{6}$ is to study the dynamics of the system by applying a driving force and measuring the resultant flux.

NEMD provides several advantages over equilibrium MD. Firstly, because the NEMD flux (signal) can be made arbitrarily larger than equilibrium fluctuations (noise) by simply increasing the driving force, the NEMD method can be made arbitrarily efficient. The only limitation is the issue 
of linearity mentioned above, but this can be controlled for by running simulations at several different field strengths. Secondly, as was pointed out by Evans and Morriss ${ }^{6}$, NEMD allows one to directly observe the microscopic physical mechanisms that are important to the transport process being simulated. Finally, NEMD simulations provide access to transport processes which do not conform to conventional transport models e.g. systems exhibiting non-linear response and spatially inhomogeneous systems.

The driving force for transport is usually generated in one of two different ways. In the boundarydriven method, high and low concentration reservoirs of the conserved quantity (energy, momentum, matter) are constructed on opposite sides of the "gradient zone". The conserved quantity flows through the gradient zone from the high concentration reservoir to the low concentration reservoir and a concentration gradient is established. Boundary-driven simulations are inherently inhomogeneous. This makes them ideally suited to direct simulation of microscopically inhomogeneous systems, such as diffusion through nanoscopic films.

In the field-driven method, an external force is applied to each particle. The external force on a particle is defined to be a function of the amount of the conserved quantity carried by that particle. As a result, the particles obey modified equations of motion which result in net transport of the conserved quantity in the absence of a gradient. The field-driven method is intrinsically homogeneous, which makes it less susceptible to finite-size effects.

The dual control volume grand canonical molecular dynamics (DCV-GCMD) method developed by Heffelfinger and van Swol ${ }^{7}$ and the similar method developed simultaneously by MacElroy ${ }^{8}$ are both examples of boundary-driven NEMD. They were designed to enable the simulation of diffusion in systems experiencing chemical potential gradients. In DCV-GCMD two local "grand canonical Monte Carlo control volumes" are set up inside the simulation volume of a molecular dynamics simulation, and grand canonical Monte Carlo (GCMC) insertions and deletions are carried out to establish different desired chemical potentials in these control volumes. The control volumes act as high and low concentration reservoirs for the diffusing species. Random exchange of energy and particles across the control volume boundaries establishes a steady-state chemical potential gradient in the gradient zone between the control volumes.

Due to the combination of Monte Carlo moves and classical Newtonian dynamics, the fluid particles in each control volume sample phase space with a distribution which resembles the grand canonical ensemble with the same temperature, volume and chemical potentials. Although the density fluctuations are generally weaker than those of the grand ensemble, the time-averages of thermodynamic properties such as species concentration have been found to agree closely with the grand ensemble values ${ }^{7}$. Similarly, the fluid particles in each small region outside of the control volumes sample from a phase space distribution which resembles the grand canonical ensemble distribution for a system with the same local temperature and chemical potentials. Several other boundary-driven schemes have been developed which do not attempt to rigorously enforce a particular thermodynamic state in the reservoirs. ${ }^{9-17}$ A review of the DCV-GCMD method, and related methods is available elsewhere. ${ }^{18}$

The DCV-GCMD has been successfully used to simulate gradient-driven diffusion in a range of different systems. ${ }^{13-17,19-30}$ However, the need to constantly insert new molecules into the control volumes limits its range of applicability, as well as that of all other boundary-driven methods. For 
dense fluids, the acceptance rate for GC insertion moves is roughly proportional to the probability of randomly inserting a particle without any overlaps. This probability decreases precipitously as the size of the inserted particle increases beyond the average particle size of the fluid. Hence the DCV-GCMD method will fail for fluid mixtures with large size ratios. Since such mixtures are too important to be ignored, we have circumvented the problem of insertion/deletion by running the simulation at constant pressure. Instead of constructing control volumes which simulate the constant $-\mu_{1} \ldots \mu_{n-1} \mu_{n} V T$ ensemble, we employ the constant- $\mu_{1} \ldots \mu_{n-1} N_{n} P T$ ensemble. This eliminates the need to exchange one of the components, allowing us to simulate diffusion in mixtures of any size-ratio.

The outline of the paper is as follows. In the next section we briefly describe the standard constant-volume DCV-GCMD method. Then we describe the new constant-pressure variant. In Section IV we present some results of the constant-pressure method for a pure equilibrium fluid in the absence of gradient. In Section V we compare the results of constant volume and constant pressure simulations of color diffusion. In Section VI we do a similar comparison for binary diffusion in a 2:1 size ratio mixture.

\section{Constant-Volume DCV-GCMD}

A comprehensive review of the DCV-GCMD and related methods has recently been published ${ }^{18}$; here we provide only a general outline. Briefly, DCV-GCMD can be thought of as a hybridization of $\mathrm{MD}$ and $\mathrm{GCMC}$ because it consists of embedding two GCMC control volumes in a standard NVT molecular dynamics simulation (Fig. 1). Each atom in the system is moved with the normal $\mathrm{MD}$ algorithm during an " $\mathrm{MD}$ phase". During this phase, the equations of motion are solved using the velocity Verlet algorithm ${ }^{31}$. Temperature control is achieved using direct velocity rescaling ${ }^{31}$. Periodically, the global average kinetic energy per particle is compared against a target value corresponding to the desired temperature. If the discrepancy exceeds some threshold value, the kinetic energy is reset to the target value by rescaling all of the particle velocities by the same factor.

After each MD phase (which proceeds for a preset number of timesteps), a "GCMC phase" follows. During the GCMC phase, a series of insertions, deletions, and (if desired) identity swaps of each component are attempted in each GCMC control volume. Destroyed atoms are simply removed from the simulation while created atoms are assigned velocities chosen on a Gaussian distribution. After the GCMC phase, the simulation proceeds with another $\mathrm{MD}$ phase, and so on.

In the spatial-decomposition parallel implementation of the method ${ }^{19,20}$, which we have used here, attempted insertions and deletions are conducted in sets of eight, corresponding to the eight subdomains of each processor domain. On each attempt, a creation or destruction (chosen with equal probability) is carried out on species $i$ (chosen randomly). If the identity swap functionality is enabled, this may be followed by an attempted change in type for a randomly selected atom of type $i$. The acceptance criteria ${ }^{7,19}$ used for attempted insertions, deletions, and identity swaps are simply those of the standard GCMC method with the system volume, $V$, number of atoms of type $i$ in the system, $N_{i}$, and the desired chemical potential of type $i$ for the system, $\mu_{i}$, replaced with the volume of $c, V(c)$, the number of atoms of type $i$ in control volume $c, N_{i}(c)$, and the desired 
chemical potential of type $i$ for control volume $c, \mu_{i}(c)$, respectively, where $c$ refers to the subdomain (see ref. 19).

Periodic boundary conditions are applied in the dimensions appropriate for the simulated system: the $x, y$, and $z$ directions for simulations of diffusion in bulk systems and through amorphous porous materials; for systems confined in a pore, the pore is situated along the $x$-axis, thus periodic boundary conditions are applied only in the $x$ direction.

The density profile, $\rho_{i}(x)$, is calculated by dividing the system volume into bins along the $x$-axis and averaging the number of atoms of type $i$ in each bin. The chemical potential profile, $\mu_{i}(x)$, is calculated using the same bins as the density profile. Outside the control volumes, the chemical potential is determined via the insertion of test atoms whereas within the control volumes the calculated energies for attempted insertions are used.

The flux of any component $i$, at any point in space $\mathrm{r}, \mathrm{J}_{i}$, is a vector quantity equal to $\rho_{i} \mathbf{u}_{i}$, where $\rho_{i}$ is the local number density of component $i$ and $u_{i}$ is the local center-of-mass velocity of component $i$. In this paper, we measure the $u_{i}$ and the $J_{i}$ relative to the fixed laboratory frame of the simulation box. In addition, we use the scalar quantity $J_{i}$ to represent the flux in the $x$-direction, since the other two components of the flux always have an average value of zero, by construction.

The flux between the two control volumes can be measured in three different ways. In the planar flux method (Fig. 1), the net movement of each species type, $i$, across a stationary plane is accumulated as the simulation progresses and used to calculate the flux. In the control volume method, the net number of particles added or subtracted (total insertions - total deletions) in control volumes $\mathrm{A}$ and $\mathrm{B}$ are accumulated and used to determine the flux for each type. In a third method, flux profiles are calculated using a binning system analogous to that used for the density profiles. For each bin, the sum of the $x$-velocities of the atoms of type $i$ is averaged over the simulation. This allows the flux of component $i$ to be calculated as a function of $x$-position.

\section{Constant-Pressure DCV-GCMD}

Because the accompanying change in energy of the insertion or deletion of large molecules is so great as to make the probability of accepting such a move prohibitively small, the range of applicability of the DCV-GCMD method as well as all other grand canonical simulation methods are limited. To overcome this problem, others have developed special incremental insertion/deletion schemes. However, these schemes can be expensive and are applicable only to particular types of molecules.

Our approach ${ }^{32}$ to solving this problem, is to avoid insertion/deletion of component $n$ altogether, so that the total the number of molecules of that component remains fixed. This amounts to performing a Legendre transform on the control volume simulations; we switch from constant$\mu_{1} \ldots \mu_{n-1} \mu_{n} V T$ to constant $-\mu_{1} \ldots \mu_{n-1} N_{n} V T$ simulations. This creates a new problem, in that the diffusion of component $n$ will cause the intensive simulation parameter $N_{n}$ in each control volume to increase or decrease. The volume $V$, which is the only other extensive simulation parameter, remains fixed, and as a result, the thermodynamic state of each control volume changes with time. To prevent this, we perform a second Legendre transform, replacing the $V$ with its intensive con- 
jugate $P$. This new constant- $\mu_{1} \ldots \mu_{n-1} N_{n} P T$ ensemble is a multicomponent analog of the isothermal-isobaric ensemble. The important feature is that $N_{n}$ is the only extensive fixed parameter. Hence as $N_{n}$ changes within a given control volume, the size of the entire control volume can change with it. The thermodynamic state is determined only by the intensive fixed parameters, and so remains unchanged.

Conventional affine expansion/contraction techniques for controlling system pressure ${ }^{31}$ can not be used here. These methods are based on global changes to the system volume, whereas we require that independent volume changes occur in the left and right control volumes. In addition, these methods would not preserve the length of the region between the two control volumes, making it impossible to define a concentration gradient. Instead, we use a type of pressure control introduced by Lupkowski and van $\mathrm{Swol}^{33}$ for equilibrium $\mathrm{MD}$ simulations of confined fluids. The Hamiltonian describing the system of interacting particles is augmented by the addition of two quasi-particles, labelled $A$ and $B$, which we will refer to as pistons. The sole purpose of the pistons is to enforce constant-pressure boundary conditions independently at opposite ends of the simulation box. For this reason, the dynamics of the pistons can be greatly simplified. Each piston has fixed $y$ and $z$ coordinates, and a fixed orientation normal to the $x$-direction. Hence, it has only one degree of freedom: translation in the $x$-direction. The Newtonian equations of motion of the augmented system are given by:

$$
\begin{aligned}
& m_{i} \overline{\mathbf{r}}_{i}=\sum_{j \neq i}^{N} \mathrm{~F}_{i j}\left(\mathbf{r}_{i j}\right)+\mathrm{F}_{i w}\left(\mathrm{r}_{i A}\right)+\mathrm{F}_{i w}\left(\mathbf{r}_{i B}\right) \\
& m_{w} \overline{\mathbf{r}}_{A}=P A_{y z} \mathbf{n}_{A}-\sum_{i=1}^{N} \mathrm{~F}_{i w}\left(\mathbf{r}_{i A}\right) \\
& m_{w} \overline{\mathbf{r}}_{B}=P A_{y z} \mathbf{n}_{B}-\sum_{i=1}^{N} \mathrm{~F}_{i w}\left(\mathbf{r}_{i B}\right)
\end{aligned}
$$

where $m_{i}$ is the mass of particle $i, \mathbf{r}_{i}$ is its position vector, and $\mathrm{F}_{i j}$ is the force on particle $i$ due to particle $j . P$ is the desired system pressure, $m_{w}$ is the piston mass, $A_{y z}$ is the cross-sectional area of the system on the $y z$ plane, $\mathbf{r}_{A}$ and $\mathbf{r}_{B}$ are the positions of the pistons and $\mathbf{n}_{A}$ and $\mathbf{n}_{B}$ are unit vectors in the positive and negative $x$-directions, respectively. $\mathbf{F}_{i w}\left(\mathbf{r}_{i w}\right)$ is the force on particle $i$ at $\mathbf{r}_{i}$ due to a piston located at $\mathbf{r}_{w}$, where $\mathbf{r}_{i w}=\mathbf{r}_{i}-\mathbf{r}_{w}$, and is given by:

$$
\mathrm{F}_{i w}(\mathbf{r})=-\frac{\mathrm{d}}{\mathrm{d} r} \phi_{i w}(r) \frac{\mathbf{r}}{r}
$$

where $\phi_{i w}(r)$ is the pair interaction energy between a particle $i$ and a piston at a distance $r$ away. It is clear from these equations, that the dynamics of the augmented system can be simulated by straight-forward extension of the standard methods of molecular dynamics simulation. In our parallel implementation, each processor calculates $\mathbf{F}_{i w}\left(\mathbf{r}_{i A}\right)$ and $\mathbf{F}_{i w}\left(\mathbf{r}_{i B}\right)$ for all the atoms which it owns. The total force on each piston is then calculated by summing over all processors. The pis- 
ton positions are updated on processor 0 , and then communicated to the other processors. In addition, the kinetic energy of the pistons is included in the temperature-rescaling algorithm.

Usually $\phi_{i w}(r)$ is chosen to be a short-ranged repulsive potential which prevents atoms from crossing through the pistons, while limiting the effect of the pistons to a layer of fluid which is only several atoms thick. The repulsive force of the fluid on the atoms is counteracted by the constant restraining force, $P A_{y z}$. As a result, once the system has equilibrated, the force exerted by the atoms on the piston will fluctuate about that of the constant restraining force, thus maintaining a constant average pressure in the $x$-direction in the fluid near the wall. As long as the system is not changing rapidly, this local pressure control will propagate throughout the system. Implicit in this approach is the assumption that the same pressure is imposed on both pistons, i.e. there is no pressure gradient in the system.

The use of the pistons in DCV-GCMD imposes certain constraints on how the simulation is conducted. Firstly, we can no longer have periodic boundaries in the $x$-direction, and so there is only one gradient zone, which passes through the center of the box. Secondly, the pistons are not allowed to penetrate the control volumes. This condition could probably be relaxed, but in our parallel implementation it simplifies the computation. Hence two buffer regions of fluid are set up between the starting positions of the pistons and the control volumes proper. As the simulation proceeds, component $n$ migrates across the simulation box, leaving one buffer region and accumulating in the other. Let us assume that this migration is from left to right. As a result, the pistons will also tend to undergo net motion from left to right, causing the left buffer to shrink and the right buffer to grow. Eventually, the left piston will reach the left control volume, at which point the simulation must be terminated. Hence the maximum duration of the simulation is limited by the size of the left-hand buffer. An additional constraint is imposed by the spatial domain decomposition. The right buffer may eventually grow to a point where it extends beyond the range of the rightmost processor domain. In this case the simulation must also be terminated. Despite the transient nature of the simulation, the two control volumes and the central gradient zone form a subsystem which is maintained at steady state, thus allowing transport properties to be measured.

\section{N. Equilibrium Molecular Dynamics using Piston Pressure Control}

As an initial test of this new constant pressure DCV-GCMD method, we carried out a comparison with the original work of Lupkowski and van Swol ${ }^{33}$, who first developed the pistons method of pressure control for confined equilibrium fluids.

We performed an equilibrium NPT molecular dynamics simulation using a pure WCA fluid. The fluid-fluid interaction potential is given by

$$
\phi_{f f}(r)= \begin{cases}4 \varepsilon_{f f}\left[\left(\frac{\sigma_{f f}}{r}\right)^{12}-\left(\frac{\sigma_{f f}}{r}\right)^{6}\right]+\varepsilon_{f f}, & r<2^{1 / 6} \sigma_{f f} \\ 0, & r \geq 22^{1 / 6} \sigma_{f f}\end{cases}
$$

where $\sigma_{f f}$ is the particle diameter and $\varepsilon_{f f}$ is a measure of the stiffness of the particle-particle repulsion. The potential is strongly repulsive at small separations, but decays smoothly to zero at the 
cut-off separation of $2^{1 / 6} \sigma$. The interaction between the fluid atoms and the piston walls ( $f w$ interaction) is defined to be the WCA analog of the 9-3 potential:

$$
\phi_{f w}(r)= \begin{cases}\varepsilon_{f w}\left[\frac{2}{15}\left(\frac{\sigma_{f w}}{r}\right)^{9}-\left(\frac{\sigma_{f w}}{r}\right)^{3}\right]+\varepsilon_{f w} \frac{\sqrt{10}}{3}, & r<(2 / 5)^{1 / 6} \sigma_{f w} \\ 0, & r \geq(2 / 5)^{1 / 6} \sigma_{f w}\end{cases}
$$

where $\sigma_{f w}$ describes the range and $\varepsilon_{f w}$ the stiffness of the repulsive potential. Note that the scalar distance variable $r$ is always positive, and so the repulsive force on the particle, defined by Eq. (4) is always directed away from the piston. The fluid-wall parameters $(f w)$ were set to those of the fluid-fluid interaction $(f)$, and all values of $\sigma$ and $\varepsilon\left(\sigma_{f f}, \sigma_{f i}, \varepsilon_{f f}\right.$, and $\left.\varepsilon_{f w}\right)$ are henceforth referred to simply as $\sigma$ and $\varepsilon$. The pressure was set to $P \sigma^{3} / \varepsilon=6.255$. The temperature was maintained at $k T /$ $\varepsilon=1.5$ using velocity rescaling. In the $x$-direction, the system was bounded by the pistons, but was free to expand and contract. In the $y$ and $z$ directions the dimensions were $12 \sigma$ and the boundary conditions were periodic. The number of atoms was fixed at 4961 , to give the same quantity of fluid per unit piston area as existed in Lupkowski's simulation, although they only used 160 atoms and had a correspondingly smaller box width of $4.31 \sigma$. The $\mathrm{MD}$ simulation was run on a $4 \times 8 \times 8$ array of processors, using a timestep of $0.005 \sigma(d / m)^{1 / 2}$. The pistons masses were set to $10 m_{f}$. The starting configuration for this NPT molecular dynamics simulation was created by equilibrating a $\mu V T$ simulation with the wall positions fixed at $\pm 6.385 \sigma$. A configuration of this system was selected which contained exactly 4961 particles. The NPT molecular dynamics simulation was then started from this configuration, with the total momentum of the system set to zero. The simulation was run for 60,000 timesteps. The piston positions were sampled every 100 timesteps and are shown in Fig. 2. The pistons fluctuated only slightly about their equilibrium positions. The system as a whole remains stationary as a result of conservation of momentum. The average separation of the walls fluctuated about a steady average value of $12.75 \sigma \pm 0.002 \sigma$. This is in close agreement with Lupkowski and van Swol's value of $12.78 \sigma$.

We also briefly examined the effect of piston mass on the simulation dynamics. As pointed out by Lupkowski. and van Swol, the piston mass should have no effect on the distribution of states sampled by the simulation, but it will affect how the simulation evolves in time. Fig. 3 shows the short-time behavior of the right piston during the first 50 timesteps of the simulation described above, in which the piston mass ratio was $m_{w} / m_{f}=10$. Also shown are the piston trajectories for mass ratios of 1 and 100. For all three mass ratios, the piston underwent oscillatory behavior with a period which was roughly proportional to $m_{w}{ }^{1 / 2}$. The amplitude of the oscillations was roughly equal in all three cases, and is determined by the initial displacement and velocity of the piston. The heaviest piston mass resulted in rather sluggish behavior, due to a slower decay of time correlations. Conversely, the lightest piston mass became unstable and failed after only a few timesteps. This was due to the characteristic timescale approaching the timestep used to integrate the equations of motion. In order to simulate this case correctly, we had to reduce the timestep by a factor of 10 .

The long-time behavior of the piston motion is shown in Fig. 4. For mass ratios of 10 and 100 the right position was plotted every 100 timesteps. The lighter piston settled down more quickly, but 
after about 3000 timesteps the trajectories look very similar. Averaged over 60,000 timesteps, the fluctuations in the wall separation were found to be $0.42 \%$ for the mass ratio of 10 and $0.38 \%$ for the mass ratio of 100 . Hence it appears that both the average properties and average fluctuations of the system are unaffected by the choice of piston mass.

To summarize, we have found that the piston mass does not significantly influence the simulation results. However, the piston mass does influence the manner in which the simulation evolves from an initial condition which is not close to the equilibrium state. The best performance is achieved using a piston mass which oscillates with a period that is about order of magnitude larger than the molecular dynamics timestep. This optimal mass should scale linearly with the system cross-sectional area, but will also depend on such factors as the isothermal compressibility of the fluid and the fluid-piston interaction potential.

\section{Color Diffusion}

In order to test the performance of the pistons in the context of gradient-driven diffusion, we applied the method to the binary system previously modeled by Heffelfinger and van Swol ${ }^{7}$. Both components were represented by the WCA potential described above. They were given identical values of $\sigma$ and $\varepsilon$, so that they differed only in their "color" or type. (That is, if we define the two fluid components as 1 and 2 and the piston as component $w$, we used

$$
\begin{aligned}
& \sigma_{11}=\sigma_{22}=\sigma_{12}=\sigma_{1 w}=\sigma_{2 w} \equiv \sigma, \text { and } \\
& \varepsilon_{11}=\varepsilon_{22}=\varepsilon_{12}=\varepsilon_{1 w}=\varepsilon_{2 w} \equiv \varepsilon \\
& m_{1}=m_{2} \equiv m_{f}
\end{aligned}
$$

for this system. The advantage of doing this type of "color diffusion" experiment is that the transport diffusivities obtained from the DCV-GCMD simulation can be compared directly with the self-diffusivity of a pure WCA fluid at the same overall density.

First, the system was simulated using a standard DCV-GCMD simulation (constant $\mu_{1}, \mu_{2}, T, V$ ), which we will refer to as the constant-volume simulation. This simulation is described in more detail elsewhere. ${ }^{18}$ The temperature was maintained at $k T / \varepsilon=1.0$ using velocity rescaling. As before, the chemical potential is specified in reduced form defined by:

$$
\mu_{i}{ }^{\prime} \equiv \mu_{i}+3 k T \ln \left(\frac{\sigma_{i}}{\Lambda_{i}}\right) .
$$

where $\mu_{i}$ is the full chemical potential, $\mu_{i}{ }^{\prime}$ is the reduced chemical potential and $\Lambda_{i}$ is the thermal de Broglie wavelength for component $i . \mu_{1}{ }^{\prime}$ and $\mu_{2}{ }^{\prime}$ were set to $1.54 \varepsilon$ and $2.34 \varepsilon$ in control volume $\mathrm{A}$ and $2.34 \varepsilon$ and $1.54 \varepsilon$ in control volume $\mathrm{B}$. Thus a concentration gradient was established, while the overall density was maintained at a constant value throughout the system.

The constant-volume simulation was carried out on an $8 \times 2 \times 2$ processor (total 32 processors). The system extended from $-24 \sigma$ to $24 \sigma$ in the $x$-direction and from $-6 \sigma$ to $6 \sigma$ in the $y$ and $z$ directions. The control volumes were located on the ranges $-18 \sigma<x<-6 \sigma$ and $6 \sigma<x<18 \sigma$. The MD timestep 
was $0.01 \sigma(m / \varepsilon)^{1 / 2}$. The simulation was started from an empty box and was equilibrated for 20,000 timesteps. After equilibration, the simulation was run for 200,000 timesteps, during which the concentration and flux profiles were sampled. The calculation was carried out on 32 nodes of an Intel Paragon MIMD parallel machine. Each timestep required about 0.1 seconds.

The control volume concentrations including density profiles and overall density agreed closely with those of Heffelfinger and van $\mathrm{Swol}^{7}$ although different simulation box dimensions were used so that the concentration gradients can not be compared directly. The average concentration gradients and fluxes are given in Table 1. The corresponding transport diffusivities in Table 1 were calculated using the relation

$$
D_{i}=-J_{i} /\left(d \rho_{i} / d x\right) \text {, }
$$

where $J_{i}$ is the flux of component $i$ in the $x$-direction. This is valid for systems which are at rest relative to the stationary reference frame. The individual estimates of transport diffusivity lay in the range $0.27 \sigma(d m)^{1 / 2}$ to $0.36 \sigma(d m)^{1 / 2}$, in close agreement with Heffelfinger and van Swol's values, which lay in the range $0.27 \sigma(d / m)^{1 / 2}$ to $0.34 \sigma(d m)^{\mathrm{C} / 2}$ ?

Having established a benchmark using constant-volume DCV-GCMD, we then simulated the same system at constant pressure using piston pressure control instead of inserting and deleting component 2 (constant- $\mu_{1} N_{2} T P$ ). We will the call this the constant-pressure simulation. The simulation was carried out on an $18 \times 4 \times 4$ processor array (total 288 processors). The simulation box extended from $-54 \sigma$ to $54 \sigma$ in the $x$-direction and from $-12 \sigma$ to $12 \sigma$ in the $y$ and $z$ directions. Note that each processor domain is a cube of size $6 \sigma$, the same as in the constant-volume simulation. However, we have doubled the system dimensions in the $y$ and $z$ directions in order to get better statistics. Also, we have extended the system in the $x$ direction by 10 processor domains, in order to provide buffer regions at each end.

The simulation was started from an empty box with fixed walls located at $\pm 36 \sigma$. To quickly equilibrate the system, the control volumes were temporarily extended out to the ends of the simulation box, and both species were inserted and deleted, using the same chemical potentials as before. Steady-state was achieved after 5,000 timesteps. At this point, the system contained 9578 species 1 atoms and 9024 species 2 atoms. From this configuration, the constant-pressure simulation was executed for 300,000 timesteps. The pressure was set at $1.4 \sigma^{3} / \varepsilon$ (equal to the average pressure from the constant-volume simulation). The piston masses were set to $3 m_{f}$. The control volumes coordinates were reset to those used in the constant-volume simulation. While the number of atoms of component 1 was allowed to vary through insertions and deletions in the control volumes to achieve a chemical potential of component 1 of 1.54 in control volume $A$ and 2.34 in control volume $B$, the number of atoms of component 2 remained fixed at 9024 . The calculation was carried out on 288 nodes of an Intel Paragon MMMD parallel machine with each timestep requiring about 0.1 seconds, the same as for the constant-volume simulation. This is to be expected, as the presence of the pistons does not add much to the computational effort, and the total number of insertions and deletions per timestep is the same for both simulations. 
The evolution of the concentration profile during consecutive 20,000 timestep intervals for component 2 is contained in Fig. 5. For clarity, all curves except the bottom one are shifted upwards in increments of $\rho \sigma^{3}=0.1$. The figure clearly demonstrates how the left buffer shrinks, the right expands, and the central portion of the simulation box does not change. The right buffer actually grows faster than the left buffer shrinks because the density of component 2 is lower on the right. . The characteristic layering of the atoms at the piston walls are somewhat apparent, although the movement of the pistons tends to blur this detail. The simulation actually terminated at timestep 291170 , when the right piston position exceeded $54 \sigma$.

The component flux profiles are shown in Fig. 6, along with predictions from a simple continuum model which will be described later. The flux profiles differ dramatically from the symmetric profiles which occurred in the constant-volume simulation (not shown) ${ }^{18}$. In the buffer zones, both components are flowing in the positive $x$-direction. This behavior is due to the bulk motion of the pistons from left to right. In the gradient zone, component 1 is diffusing strongly, in the negative $x$-direction, while the flux of component 2 is roughly constant throughout the box. This raises two important questions:

- How can the diffusion coefficient be determined from the flux and concentration profiles?

-What factors control the fluxes in the various parts of the simulation box?

We can best answer the former question by first addressing the latter. First of all, we need to replace the absolute fluxes $J_{i}$ by the more general diffusive fluxes $J_{i D}$, which are defined relative to the molar average velocity ${ }^{34}$

$$
J_{i D}=J_{i}-x_{i} \sum_{i=1}^{n} J_{i},
$$

where $x_{\mathrm{i}}$ is the mole fraction of component $i$. In this case, Fick's Law is written as

$$
\frac{d \rho_{i}}{d x}=-\frac{J_{i D}}{D_{i}}=\frac{J_{t o t}}{D_{i} \rho_{t o t}} \rho_{i}-\frac{J_{i}}{D_{i}} .
$$

In general, Eq. (13) can not be solved exactly. However, in the case of color diffusion, where the diffusion coefficients and total density do not vary with position, the equation can be solved analytically. The solution is an exponential profile,

$$
\left(\frac{\rho_{i}(x)-\rho_{i}^{\infty}}{\rho_{i}^{A}-\rho_{i}^{\infty}}\right)=\left(\frac{\rho_{i}^{B}-\rho_{i}^{\infty}}{\rho_{i}^{A}-\rho_{i}^{\infty}}\right)^{\frac{x-x_{A}}{x_{B}-x_{A}}},
$$

where 


$$
\rho_{i}^{\infty} \equiv \frac{J_{i} \rho_{t o t}}{J_{t o t}}
$$

For zero total flux (which is the case for the constant-volume color diffusion ${ }^{18}$ ), the parameters $\rho_{i}^{\infty}$ are infinite and Eq. (14) becomes linear. However, in general, the $\rho_{i}^{\infty}$ are finite, resulting in non-linear concentration profiles. The $\rho_{i}^{\infty}$ are determined by some external constraint on the system, which in the case of our simulation is the requirement that momentum be conserved. This is one example of the general concept of the bootstrap condition in diffusion experiments. ${ }^{34}$ The full momentum balance is a little complicated in the piston simulation, but we can simplify it by making assumptions which are reasonably accurate for slowly diffusing systems:

1. The simulation is in a quasi-steady state, in that the "macroscopic" descriptors of the system change much more slowly than local fluctuations in density, momentum, etc.

2. The simulation box is divided into three distinct regions: the left buffer and control volume $A$, with total length $L_{A}$, the gradient zone, of length $L_{G}$, and the right buffer and control volume $B$, with total length $L_{B}$. These dimensions are treated as constant.

3. The fluxes do not change with time (steady-state).

4. The flux of component two $\left(J_{2}\right)$ is constant throughout the simulation box (steady-state).

5. The concentrations of the two components are constants in the left control volume and buffer zone $\left(\rho_{i}^{A}\right)$ and in the right control volume and buffer zone $\left(\rho_{i}^{B}\right)$.

6. As a consequence of assumptions 4 and 5 , the flux of component one is constant in the left control volume and buffer zone $\left(J_{\mathrm{I}}{ }^{A}\right)$ and in the right control volume and buffer zone $\left(J_{1}{ }^{B}\right)$.

7. Insertion and deletion of component 1 has no net effect on the momentum of the system. In fact, the effect should be to add momentum in the opposite direction to the diffusion of component 1 , since particles are added with zero momentum on average but particles which are removed will tend to be moving in the direction of $J_{1}$.

With these assumptions, the conservation of momentum constraint results in the following expression for $J_{2}$ in a piston simulation involving particles of different mass.

$$
J_{2}=\frac{\left(m_{1} J_{1}^{G}+m_{2} J_{2}\right) L_{G}}{\frac{P_{t o r}}{A J_{2}}-\frac{L_{A}}{\rho_{2}^{A}}\left(m_{1} \rho_{1}^{A}+m_{2} \rho_{2}^{A}\right)-\frac{L_{B}}{\rho_{2}^{B}}\left(m_{1} \rho_{1}^{B}+m_{2} \rho_{2}^{B}\right)} .
$$

$P_{t o t}$ is the total linear momentum in the $x$ direction, $A$ is the cross-sectional area of the box, and $m_{1}$ and $m_{2}$ are the masses of the two components. In the case of the color diffusion simulation, where the particles are of equal mass, and the total momentum is zero, this equation simplifies to an explicit expression for $\rho_{2}^{\infty}$. 


$$
\rho_{2}^{\infty} \equiv \frac{J_{2} \rho_{t o t}}{J_{t o t}^{G}}=-\frac{L_{G}}{L_{A} / \rho_{2}^{A}-L_{B} / \rho_{2}^{B}} .
$$

The physical meaning of this equation is that the motion of the system from left to right due to the diffusion of component 2 must be balanced by an opposing motion from right to left within the gradient zone, due to the diffusion of component 1 . The smaller the gradient zone, relative to regions $A$ and $B$, the larger must be the flux. Hence, in the limit of very large buffer zones, the mass of the gradient zone becomes negligible, and the buffer zones must have zero flux. In this limit, the pistons do not move. In fact, the only non-zero flux is that of component 1 in the gradient zone. Conversely, in the limit of a very large gradient zone, the mass of the regions $A$ and $B$ becomes negligible, and the gradient zone has zero net flux. In this limit, the pistons undergo the fastest possible motion from left to right. Hence we see that increasing the buffer regions extends the duration of a simulation in two ways. Firstly, it allows the pistons to move a larger distance. Secondly, it reduces the rate of motion.

Differentiating Eq. (14) and inserting in Eq. (13), we obtain an explicit expression for the flux of component 2:

$$
J_{2}=\frac{D_{2} \rho_{2}^{\infty}}{L_{G}} \ln \left[\frac{\rho_{2}^{A}-\rho_{2}^{\infty}}{\rho_{2}^{B}-\rho_{2}^{\infty}}\right] .
$$

From Eq. (15) it follows that

$$
J_{1}^{G}=J_{2}\left(\frac{\rho_{t o t}}{\rho_{2}^{\infty}}-1\right) .
$$

Finally, the absence of a concentration gradients in regions $A$ and $B$ requires that the diffusion fluxes vanish, and so it follows from Eq. (12) that

$$
\begin{aligned}
& J_{1}^{A}=J_{2}\left(\frac{\rho_{t o t}}{\rho_{2}^{A}}-1\right), \text { and } \\
& J_{1}^{B}=J_{2}\left(\frac{\rho_{t o t}}{\rho_{2}^{B}}-1\right) .
\end{aligned}
$$

In order to compare these predictions for the fluxes against the simulation fluxes, we need to assign values to the parameters $L_{A}, L_{B}, L_{G}, \rho_{t o t}, \rho_{2}{ }^{A}, \rho_{2}{ }^{B}$ and $D_{2}$. The value of the diffusion coefficient was estimated by a method described below. Other parameters were taken directly from the constant-pressure simulation and are summarized in Table 2 . The resultant fluxes are plotted in Fig. 6, together with the original simulation data. The fluxes in the three different regions are in qualitative agreement with the simulation data, although quantitative agreement was not achieved. This is to be expected, given the many simplifying assumptions, but it validates the 
underlying principle of the model, which is that the evolution of the simulation is controlled by Fick's law and conservation of momentum.

The continuum analysis provides us with the answer to our first question. Eq. (12) provides a local definition of the diffusion coefficients, but it requires the local concentration gradient and local diffusion flux, both of which depend on position. Of course, since the overall density of the system is constant, we expect that the local diffusion coefficient turns out to be independent of position. In order to carry out this analysis, we performed a three-parameter least-squares fit of the concentration profiles in the gradient zone to the exponential form of Eq. (14), and then calculated the concentration gradients analytically. The resulting profiles are shown in Fig. 7, along with the diffusion fluxes calculated from Eq. (11). It can be seen that the concentration gradients and diffusion fluxes decrease in magnitude across the gradient zone. The resultant diffusion coefficients are shown in Fig. 8. There is a significant decrease in the diffusion coefficients from left to right. This is partly due to the statistical error in the least-squares fit to the concentration profiles. By fitting the density profiles so as to minimize the square relative error of the density plus the standard deviation of the diffusion coefficient, a very similar fit is obtained, but with a uniform diffusion coefficient profile. The average diffusion coefficients are very close in either case. The average values for the straight least squares fit are $D_{1}(m / \varepsilon)^{1 / 2} / \sigma=0.357$ and $D_{2}(m / \varepsilon)^{1 / 2} /$ $\sigma=0.366$. The average of these two numbers was used in the continuum model described above.

In Table 3 the diffusion coefficients calculated using this simplified analysis as well as those obtained from the least-squares fit to the concentration profiles are compared with those of the constant-volume color diffusion simulation.

The diffusion coefficients obtained using the simplified and more detailed analyses of the constant-pressure simulation agree well with each other. However, they were somewhat higher than those from the constant-volume simulations. There are several possible reasons for this. Firstly, the total density in the constant-pressure simulation gradient zone was slightly lower (ca. 1\%) than in the constant volume simulations, which should raise the diffusivity by about $1.5 \%$, based on the correlation of Ruckenstein ${ }^{35}$. Secondly, there are significant statistical errors in the diffit sivities from both methods, which are difficult to estimate precisely, but are probably on the order of several percent. Hence the observed differences are probably not statistically significant.

\section{Binary Diffusion}

As a second test of the constant-pressure method, we applied it to a fairly dense binary mixture of WCA spheres, with a size ratio of $1: 2$, i.e. $\sigma_{1}=\sigma, \sigma_{2}=2 \sigma$. The Lennard-Jones energy $\varepsilon$ and mass $m$ of both components were the same. As before, both constant volume and constant pressure simulations were performed. The reduced temperature was set to $k T=1$. The chemical potentials used in the constant volume simulation, as well as the pressure used in the constant pressure simulation are given in Table 4. These values were chosen to create the following conditions. A volume fraction of 0.3 in control volume $\mathrm{A}$ was targeted, with $30 \%$ component 1 by volume. The volume fraction in control volume $\mathrm{B}$ was chosen to match the pressure in control volume $\mathrm{A}$, with $70 \%$ component 2 by volume. The required volume fraction in control volume $B$ turned out to be 0.22 . The chemical potentials required to create these conditions were calculated using the CamahanStarling-Mansoori equation of state for hard sphere mixtures. ${ }^{36}$ Effective hard-sphere diameters 
for the WCA particles were calculated using a correlation due to Ben-Amotz ${ }^{35,37}$ The final densities differed from the target values by less than $2 \%$.

The constant-volume simulation was performed on an $8 \times 4 \times 4$ array of 128 processors. The system extended from $-24 \sigma$ to $24 \sigma$ in the $x$-direction and from $-12 \sigma$ to $12 \sigma$ in the $y$ and $z$ directions. The control volumes were located on the ranges $-18 \sigma<x<-6 \sigma$ and $6 \sigma<x<18 \sigma$. The $\mathrm{MD}$ timestep was $0.005 \sigma(m / \varepsilon)^{1 / 2}$. The simulation was started from an empty box and was equilibrated for 500,000 timesteps. After equilibration, the simulation was run for 3,300,000 timesteps, during which the concentration, chemical potential and flux profiles were sampled. The relatively long duration of the simulation was required in order to obtain very accurate measurements of the chemical potential profiles of both components. The average acceptance rates for attempted insertions were 0.1 and 0.001 for components 1 and 2 respectively. To improve the exchange of component 2, a 10\%/ $90 \%$ bias was used when selecting which component to insert or delete. Attempts to use the constant volume method to simulate mixtures with a total volume fraction of 0.5 failed due to extremely low acceptance rates for the large component. The calculation was carried out on 128 nodes of Sandia's Intel Teraflop MIMD parallel machine with each timestep requiring about 0.03 seconds.

The constant-pressure simulation was carried out on a $14 \times 4 \times 4$ array of 224 processors in a manner similar to that of the constant-pressure color diffusion simulation described in the previous section. The simulation box extended from $-42 \sigma$ to $+42 \sigma$ in the $x$-direction and from $-12 \sigma$ to $+12 \sigma$ in the $y$ and $z$ directions. The simulation was started from an empty box with fixed walls located at $-40 \sigma$ and $+19 \sigma$. These positions were chosen to be slightly greater than the lowest allowable $x$-coordinates for the left and right pistons, so as to maximize the available range of leftto-right motion, while giving the pistons some room to fluctuate during start-up. To quickly equilibrate the system, the control volumes were temporarily extended out to the ends of the simulation box, and both species were inserted and deleted, using the same chemical potentials as before. A stable concentration profile was established 75,000 timesteps. From this configuration, a short constant pressure run was conducted, in order to estimate the rate of motion of the pistons. Then a constant-pressure simulation was executed for 300,000 timesteps. The pressure was set at $P \sigma^{3} /$ $\varepsilon=0.823$ (equal to the average pressure from the constant-volume simulation). The control volumes coordinates were reset to those used in the constant-volume simulation. The calculation was carried out on 224 nodes of Sandia's Intel Teraflop MIMD parallel machine with each timestep requiring about 0.03 seconds, the same as for the constant-volume simulation.

In Fig. 9 we compare the concentration profiles obtained from the two simulations. The agreement in control volume A is good, but in control volume $\mathrm{B}$, the constant-pressure simulation seems to have a small gradient. This is probably due to an insufficient rate of insertion of component 1 , which is constantly diffusing out of the left side of control volume $B$

The flux profiles from both simulations are shown in Fig. 10. The constant-volume simulation exhibits an asymmetry in the fluxes; component 1 diffuses nearly ten times faster than component 2 . The perfect symmetry introduced by the periodic boundary conditions allow the two components to diffuse at different rates without violating the zero total momentum constraint. Instead, the momentum of each component sums to zero; the average value of the component fluxes is only about $1 \%$ of the value in either gradient zone. The constant-pressure fluxes are qualitatively 
similar to those from the constant-pressure color diffusion simulation. The flux of component 2 is small and positive throughout. The flux of component 1 is large and negative in the gradient zone, and positive on the left and right. The average total flux is slightly negative, but only about $2 \%$ of the flux of component 1 in the gradient zone.

The local binary diffusion coefficients were calculated by the detailed method described in the previous section and the resultant $D_{i}$ profiles are shown in Fig. 11. The agreement between the two simulation methods is excellent. $D_{1}$ is smaller than $D_{2}$ by a factor of 4 , which is a consequence of the fact that Eq. (13) defines the $D_{i}$ in terms of number density gradient. It should also be noted that both $D_{1}$ and $D_{2}$ depend quite strongly on position, i.e. composition. Both of these characteristics, asymmetry and composition-dependence make binary diffusion coefficients less than ideal descriptors of multicomponent diffusion.

The Maxwell-Stefan diffusion coefficients are defined in terms of the true driving forces for diffusion. As a result, they exhibit less concentration dependence, and are symmetric, if the GibbsDuhem relation is satisfied. For a binary system, the Maxwell-Stefan diffusion coefficients are defined by ${ }^{34}$ :

$$
\begin{aligned}
& J_{1 D}=-D_{12} \frac{\rho_{1}}{k T} \frac{\mathrm{d} \mu_{1}}{\mathrm{~d} x}, \text { and } \\
& J_{2 D}=-D_{21} \frac{\rho_{2}}{k T} \frac{\mathrm{d} \mu_{2}}{\mathrm{~d} x} .
\end{aligned}
$$

The factors multiplying the $D_{i j}$ on the right hand side are the fundamental driving forces for diffusion; the system is at equilibrium only when they vanish. In order to estimate the $D_{i j}$, we first need to evaluate these forces. The chemical potential profiles of component 1 and 2 obtained from the constant-volume simulation, as well as the profile of component 1 from the constant pressure simulation are shown in Fig. 12. The profiles for component 1 from both simulation methods differ slightly. This is consistent with the slightly lower concentration profile in the constant pressure simulation (see Fig. 9). The levels of statistical variation from both methods are very similar. The constant volume simulation was run ten times longer than the constant pressure simulation, but Widom insertion were performed on component 1 only $10 \%$ of the time, hence the overall sample sizes used for the chemical potential profile of component 1 in both simulations are about equal.

In order to estimate the local chemical potential gradient, we divided the profiles shown in Fig. 12 into six pieces, and performed a linear least-squares fit to estimate the gradient at the mid-point of each piece. Standard errors on the gradient measurements were estimated in the usual way from the scatter of the data about the least-squares fit. The resultant driving forces are shown in Fig. 13. We have also included the data from the second gradient zone, which passes through the periodic boundary. The driving forces clearly decrease in magnitude in going from control volume $A$ to control volume B. A similar decrease occurs in the diffusion fluxes (not shown). The total driving force shows no systematic variation, but rather appears to fluctuate randomly about zero. The error bars shown are based on the standard errors from the least squares fits, and provide only a lower bound on the total uncertainty in these data points. We conclude that the driving forces at 
any given point are approximately zero. This is in agreement with the Gibbs-Duhem relation, which for a binary mixture at constant temperature and pressure reduces to:

$$
\rho_{1} d \mu_{1}+\rho_{2} d \mu_{2}=0
$$

The fact that the Gibbs-Duhem relation is satisfied is strong evidence that local equilibrium exists at all points in the gradient zone, despite the presence of fairly large fluxes.

The driving forces and the diffusion fluxes, calculated as before from Eq. (12), were used to calculate the $D_{i j}$ via Eq. (22). The resultant profiles for $D_{12}$ and $D_{21}$ are shown in Fig. 14 and Fig. 15 respectively. The error bars are standard error estimates based on the standard errors from the least squares fits to the chemical potential profiles. These error bars are also consistent with the variation amongst points in a given profile, assuming that the points are independent samples from the same distribution. None of the profiles show any significant positional dependence. The average values of the diffusion coefficients are shown in Table 5 . The agreement between the constant volume and constant pressure simulations is not as good as it was for the binary diffusion coefficients. This is due in part to the greater statistical uncertainty in the chemical potential profiles.

\section{Conclusions}

We have shown that constant-pressure DCV-GCMD enables the simulation of gradient-driven diffusion in dense fluids containing one large-molecule non-insertable component. Furthermore, we have demonstrated how appropriate transport coefficients can be extracted from such a simulation by the use of local definitions for the diffusion fluxes and concentration or chemical potential gradients. Finally we have verified the existence of local equilibrium in a binary mixture undergoing steady-state gradient-driven diffusion.

The only drawback of the method is that the duration of the simulation is limited by the initial size of the buffer zone constructed at the high concentration boundary. However, the method enables us to simulate gradient-driven diffusion in many systems which would otherwise be impossible. In addition, the method can be extended to other ensembles, providing access to a wide variety of systems. For example, in order to simulate a multicomponent liquid mixture of large molecules, the semigrand ensemble of Kofke and Glandt ${ }^{38}$ could be used. Constant temperature, pressure, and total number of molecules, as well as their relative chemical potentials would be imposed in the control volumes. This would require identity swap Monte Carlo moves between components (isomerizations), but would not require insertion of any molecules (except during initialization). For large molecules with similar structures, identity swap moves will have a much higher acceptance rate than insertion moves.

Potential applications of the constant-pressure DCV-GCMD method include diffusion of drug molecules across biological membranes. A semi-grand extension of the method could be used to simulate transport of large molecules in nanoporous materials under non-zero loading conditions (e.g. selective transport and sorption of xylene isomers in faujasite). 


\section{REFERENCES}

1. S. R. de Groot and P. Mazur, Non-Equilibrium Thermodynamics, Interscience, New York, (1962).

2. D. G. Miller, V. Vitagliano, and R. Sartorio, J. Phys. Chem., 90 1509, (1986).

3. H.J.V. Tyrrell and K. R. Harris, Diffusion in Liquids, Butterworths, London, (1984).

4. J. M. D. McElroy, in Diffusion in Polymers, P. Neogi Ed., Marcel-Dekker, (1996)

5. S. C. Ying, I Vattulainen, J. Merikoski, T. Hjelt, and T. Ala-Nissila, Phys. Rev. B, 58, 2170 (1998)

6. D. J. Evans and G. P. Morriss, Statistical Mechanics of Non-Equilibrium Liquids, Academic Press (1990)

7. G. S. Heffelfinger and F. van Swol, J. Chem. Phys., 100, 7548 (1994).

8. J. M. D. MacElroy, J. Chem. Phys., 101, 5274 (1994).

9. J. H. Thurtell and G. W. Thurtell, J. Chem. Phys., 88, 6641 (1988).

10. W. Dong and H. Luo, Physical Review E, 52, 801 (1995).

11. J. J. Erpenbeck and W. W. Wood, in Statistical Mechanics, Part B: Time-dependent Processes, edited by B. J. Berne, Modern Theoretical Chemistry Vol. 6 (Plenum, New York, 1977).

12. S. Fritzsche, R. Haberlandt, and J. Kärger, Zeitschrift für Physikalische Chemie, 189, 221 (1995).

13. J. W. Evans, M. H. Abbasi, and A. Sarin, J. Chem. Phys., 72, 2967 (1980).

14. D. M. Smith, AIChE Journal, 32, 329 (1986).

15. M. Sun and C. Ebner, Phys. Rev. A, 46, 4813 (1992).

16. D. M. Ford and E. D. Glandt, J. Phys. Chem., 99, 11543 (1995).

17. S. K. Kjelstrup and B. Hafskjold, Ind. Eng. Chem. Res., 35, 4203 (1996).

18. A. P. Thompson, D. M. Ford and G. S. Heffelfinger, J. Chem. Phys., 109, 6406 (1998).

19. G. S. Heffelfinger and D. M. Ford, Mol. Phys., 94, 659 (1998).

20. D. M. Ford and G. S. Heffelfinger, Mol. Phys., 94, 673 (1998).

21. G. S. Heffelfinger, P. I. Pohl, and L. J. D. Frink, Mat. Res. Soc. Symp. Proc., 366, 225 (1995).

22. P. I. Pohl, G. S. Heffelfinger, and D. M. Smith, Mol. Phys. 89, 1725 (1996).

23. P. I. Pohl and G. S. Heffelfinger, Mat. Res. Soc. Symp. Proc., 464, 339 (1997).

24. S. Furukawa, T. Shigeta, and T. Nitta, J. Chem. Engr. of Japan, 29, 725 (1996).

25. S. Furukawa, and T. Nitta, J. Chem. Engr. of Japan, 30, 116 (1997).

26. R. F. Cracknell, D. Nicholson, and N. Quirke, Phys. Rev. Lett., 74, 2463 (1995).

27. D. Nicholson, R. Cracknell, and N. Quirke, Langmuir, 12, 4050 (1996).

28. S. Sunderrajan, C. K. Hall, B. D. Freeman, J. Chem. Phys., 105, 1621 (1996). 
29. D. M. Ford and G. S. Heffelfinger, American Physical Society Annual Meeting, Kansas City, MO, 17-21 March (1997)

30. L. Xu, M. G. Sedigh, M. Sahimi, and T. T. Tsotsis, Phys. Rev. Lett., 80, 3511, (1998)

31. M. P. Allen and D. J. Tildesley, Computer Simulation of Liquids, (Clarendon Press, Oxford, 1987).

32. F. van Swol and G. S. Heffelfinger, Mat. Res. Soc. Symp. Proc., 408, 299 (1996).

33. M. Lupkowski and F. van Swol, J. Chem. Phys., 93, 737 (1990).

34. R. Taylor, and R. Krishna, Multicomponent Mass Transfer, (Wiley, 1993).

35. E. Ruckenstein and H. Liu, Ind. Eng. Chem. Res., 36, 3927 (1997)

36. T. M. Reed and K. E. Gubbins, Applied Statistical Mechanics, Butterworth-Heinemann (1991)

37. D. Ben-Amotz and D. R. Herschbach, J. Phys. Chem., 94, 4714 (1990)

38. D. A. Kofke and E. D. Glandt, Mol. Phys., 64, 1105 (1988) 


\section{Figure Captions}

Figure 1. A schematic of a DCV-GCMD simulation. Two control volumes, $A$ and $B$, are used for insertions/deletions to achieve spatial chemical potential control of each species in the system.

Figure 2. Plot of piston positions versus time for an NPT molecular dynamics simulation of a pure WCA fluid. The temperature was $1.5 \& k$, the pressure was $6.255 \& \sigma^{3}$. The lines are (from bottom) the left piston position, the right piston position, and the piston separation.

Figure 3. Short-time piston dynamics. Plot of the right piston position versus time for the first 50 timesteps, for $m_{w} / m_{f}$ ratios of 1 (diamonds), 10 (squares), and 100 (triangles). The fourth curve (circles) used a mass ratio of 1 , but with the $\mathrm{MD}$ timestep reduced by a factor of 10 , and only every tenth point plotted. All four simulations have the same starting point as the simulation shown in Fig. 2.

Figure 4. Long-time piston dynamics. Plot of right piston position versus time at 100 timestep intervals for $m_{w} / m_{f}$ ratios of 10 (squares), and 100 (triangles). The second curve is shifted upwards by $0.2 \sigma$ for clarity. Both simulations have the same starting point as the simulation shown in Fig. 2.

Figure 5. Time evolution of the concentration profile for component 2 in the constant-pressure DCV-GCMD simulation. Each line represents the average concentration profile accumulated over 20,000 timesteps; plot labels indicate the last timestep sampled. For clarity all the lines except the lowest have been offset vertically by successive increments of $\rho_{2} \sigma^{3}=0.1$. The grey lines represent the boundaries of the control volumes.

Figure 6. Average flux profiles sampled between timesteps 100,000 and 200,000 of the constantpressure DCV-GCMD simulation. The open squares are for component 1 and the filled squares are for component 2 . The fluxes are measured relative to the "stationary coordinate reference frame", i.e. the simulation box coordinates. The solid lines are the predictions of the continuum model described in the text. The grey lines represent the boundaries of the control volumes.

Figure 7. Plot of concentration gradient profiles (circles, left axis) and diffusion flux profiles (triangles, right axis) in the gradient zone from the constant-pressure color diffusion simulation. The open symbols are for component 1 and the filled symbols are for component 2 .

Figure 8. Diffusion coefficient profiles for components 1 (open circles) and 2 (filled circles) from the constant-pressure color diffusion simulation.

Figure 9. Concentration profiles from simulations of gradient-driven diffusion in a binary mixture of WCA spheres with a 1:2 size ratio. The open symbols are for component 1 (left axis) and the filled symbols are for component 2 (right axis). The circles are from the constant volume simulation, and squares are from the constant pressure simulation. The grey lines represent the boundaries of the control volumes.

Figure 10. Flux profiles from simulations of gradient-driven diffusion in a binary mixture of WCA spheres with a 1:2 size ratio. The open symbols are for component 1 (left axis) and the filled symbols are for component 2 (right axis). The circles are from the constant volume simula- 
tion, and squares are from the constant pressure simulation. The grey lines represent the boundaries of the control volumes.

Figure 11. Binary diffusion coefficient profiles for the binary system of WCA spheres with a 1:2 size ratio. The open symbols are for component 1 and the filled symbols are for component 2 . The circles are from the constant volume simulation (central gradient zone), and squares are from the constant pressure simulation.

Figure 12. Chemical potential profiles for the binary system of WCA spheres with a 1:2 size ratio. The open symbols are for component 1 (left axis) and the filled symbols are for component 2 (right axis). The circles are from the constant volume simulation (central gradient zone), and squares are from the constant pressure simulation (component 1 only).

Figure 13. Profiles of the thermodynamic driving forces from the constant volume simulation of the binary system of WCA spheres with a 1:2 size ratio. The open circles are for component 1 and the closed circles are for component 2 . The triangles are the sum of the two forces. The solid lines are the profiles in the central gradient zone; the dashed lines are the profiles from the periodic gradient zone (with the $x$ coordinate shifted and reversed appropriately). The error bars are based on standard error estimates from the piecewise linear fits used to calculate the local chemical potential gradients.

Figure 14. Maxwell-Stefan diffusion coefficient profiles for component 1 in the binary system of WCA spheres with a 1:2 size ratio. The circles are from the constant-volume simulation, with the solid line indicating the central gradient zone and the dashed line indicating the periodic gradient zone (with the $x$ coordinate shifted and reversed appropriately). The squares are from the constant-pressure simulation. The error bars are based on standard error estimates from the piecewise linear fits used to calculate the local chemical potential gradients.

Figure 15. Maxwell-Stefan diffusion coefficient profiles for component 2 in the binary system of WCA spheres with a 1:2 size ratio. All data are from the constant-volume simulation, with the solid line indicating the central gradient zone and the dashed line indicating the periodic gradient zone (with the $x$ coordinate shifted and reversed appropriately). The error bars are based on standard error estimates from the piecewise linear fits used to calculate the local chemical potential gradients. 
Table 1: Concentration gradients, absolute fluxes and resultant diffusion coefficients from the constant-volume DCV-GCMD color diffusion simulation.

\begin{tabular}{|c|c|c|c|c|c|}
\hline \multirow{2}{*}{ Component } & \multirow{2}{*}{$\sigma^{4} d \rho_{i} / d x$} & \multicolumn{2}{|c|}{ Control Volume } & \multicolumn{2}{c|}{ Flux Plane } \\
\cline { 3 - 6 } & & $J_{i} \sigma^{3}(\mathrm{~m} / \varepsilon)^{1 / 2}$ & $D_{i}(\mathrm{~m} / \varepsilon)^{1 / 2} / \sigma$ & $J_{i} \sigma^{3}(\mathrm{~m} / \varepsilon)^{1 / 2}$ & $D_{i}(\mathrm{~m} / \varepsilon)^{1 / 2} / \sigma$ \\
\hline 1 & 0.0124 & 0.00447 & 0.360 & 0.00451 & 0.364 \\
\hline 2 & 0.0122 & 0.00331 & 0.271 & 0.00334 & 0.274 \\
\hline Average & 0.0123 & 0.00389 & 0.314 & 0.00393 & 0.319 \\
\hline
\end{tabular}

Table 2: Parameters used in the continuum model of the constant-pressure color diffusion simulation.

\begin{tabular}{|c|c|c|c|c|c|c|}
\hline$D_{2}(m / \varepsilon)^{1 / 2} / \sigma$ & $\sigma^{3} \rho_{2}^{A}$ & $\sigma^{3} \rho_{2}^{B}$ & $\sigma^{3} \rho_{t o t}$ & $L_{A} / \sigma$ & $L_{B} / \sigma$ & $L_{G} / \sigma$ \\
\hline 0.362 & 0.30 & 0.15 & 0.45 & 30 & 30 & 12 \\
\hline
\end{tabular}

Table 3: Concentration gradients, diffusion fluxes and resultant diffusion coefficients from the constant-volume and constant pressure color diffusion simulations.

\begin{tabular}{|c|c|c|c|c|c|c|}
\hline & \multicolumn{3}{|c|}{ Constant Volume DCV-GCMD } & \multicolumn{3}{|c|}{ Constant Pressure DCV-GCMD } \\
\hline Spec. & $\sigma^{4} \frac{\mathrm{d} \rho_{i}}{\mathrm{~d} x}$ & $J_{i D} \sigma^{3} \sqrt{\frac{m}{\varepsilon}}$ & $\frac{D_{i}}{\sigma} \sqrt{\frac{m}{\varepsilon}}$ & $\sigma^{4} \frac{\mathrm{d} \rho}{\mathrm{d} x}$ & $J_{i D} \sigma^{3} \sqrt{\frac{m}{\varepsilon}}$ & $\frac{D_{i}}{\sigma} \sqrt{\frac{m}{\varepsilon}}$ \\
\hline 1 & 0.0124 & 0.00394 & 0.317 & 0.0106 & 0.00399 & 0.357 \\
\hline 2 & 0.0122 & 0.00394 & 0.323 & 0.0109 & 0.00399 & 0.366 \\
\hline Ave. & 0.0123 & 0.00394 & 0.320 & 0.0108 & 0.00399 & 0.362 \\
\hline
\end{tabular}

Table 4: Thermodynamic conditions used in binary diffusion simulations.

\begin{tabular}{|c|c|c|c|c|c|c|}
\hline & $\mu_{1}^{A} / \varepsilon$ & $\mu_{1}{ }^{B} / \varepsilon$ & $\mu_{2}^{A} / \varepsilon$ & $\mu_{2}{ }^{B} / \varepsilon$ & $P \sigma^{3} / \varepsilon$ & $k T / \varepsilon$ \\
\hline Constant $\mathrm{V}$ & 1.007 & 1.267 & 8.886 & 7.219 & - & 1.0 \\
\hline Constant $\mathrm{P}$ & 1.007 & 1.267 & - & - & 0.823 & 1.0 \\
\hline
\end{tabular}


Table 5: Average binary and Maxwell-Stefan diffusion coefficients from the constant-volume and constant pressure simulations of the 2:1 size ratio mixture.

\begin{tabular}{|c|c|c|c|c|}
\hline & $\frac{D_{1}}{\sigma} \sqrt{\frac{m}{\varepsilon}}$ & $\frac{D_{2}}{\sigma} \sqrt{\frac{m}{\varepsilon}}$ & $\frac{D_{12}}{\sigma} \sqrt{\frac{m}{\varepsilon}}$ & $\frac{D_{21}}{\sigma} \sqrt{\frac{m}{\varepsilon}}$ \\
\hline Constant Volume & 0.104 & 0.368 & $0.25 \pm 0.01^{\mathrm{a}}$ & $0.25 \pm 0.01^{\mathrm{a}}$ \\
\hline Constant Pressure & 0.101 & 0.358 & $0.20 \pm 0.01^{\mathrm{a}}$ & - \\
\hline
\end{tabular}

a. Uncertainty estimates are based on the standard errors from the piecewise linear fits to the chemical potential profiles. 
Figure 1. A schematic of a DCV-GCMD simulation. Two control volumes, $A$ and $B$, are used for insertions/deletions to achieve spatial chemical potential control of each species in the system.

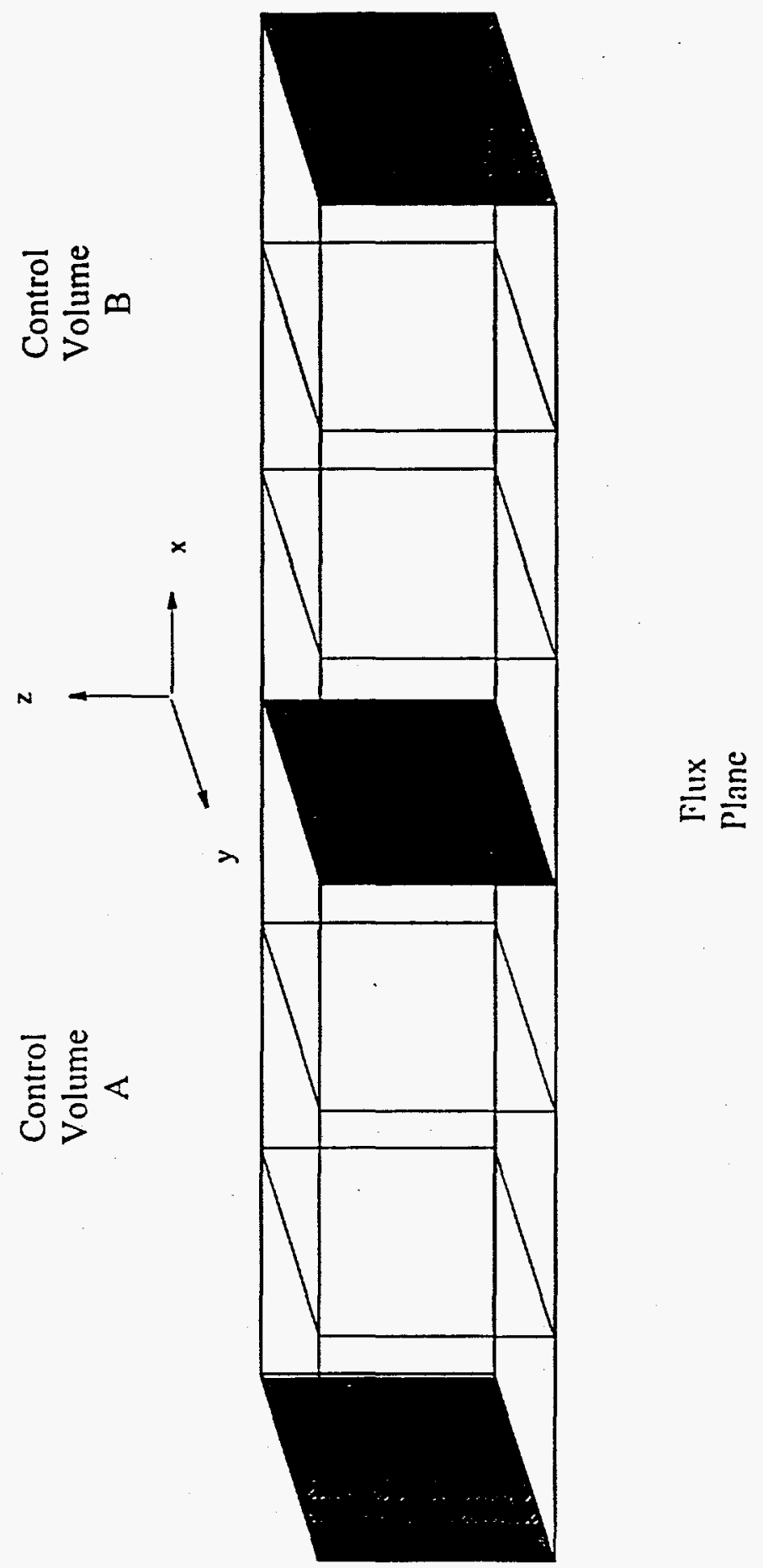


Figure 2. Plot of piston positions versus time for an NPT molecular dynamics simulation of a pure WCA fluid. The temperature was $1.5 \mathrm{~g} k$, the pressure was $6.255 \mathrm{~d} \sigma^{3}$. The lines are (from bottom) the left piston position, the right piston position, and the piston separation.

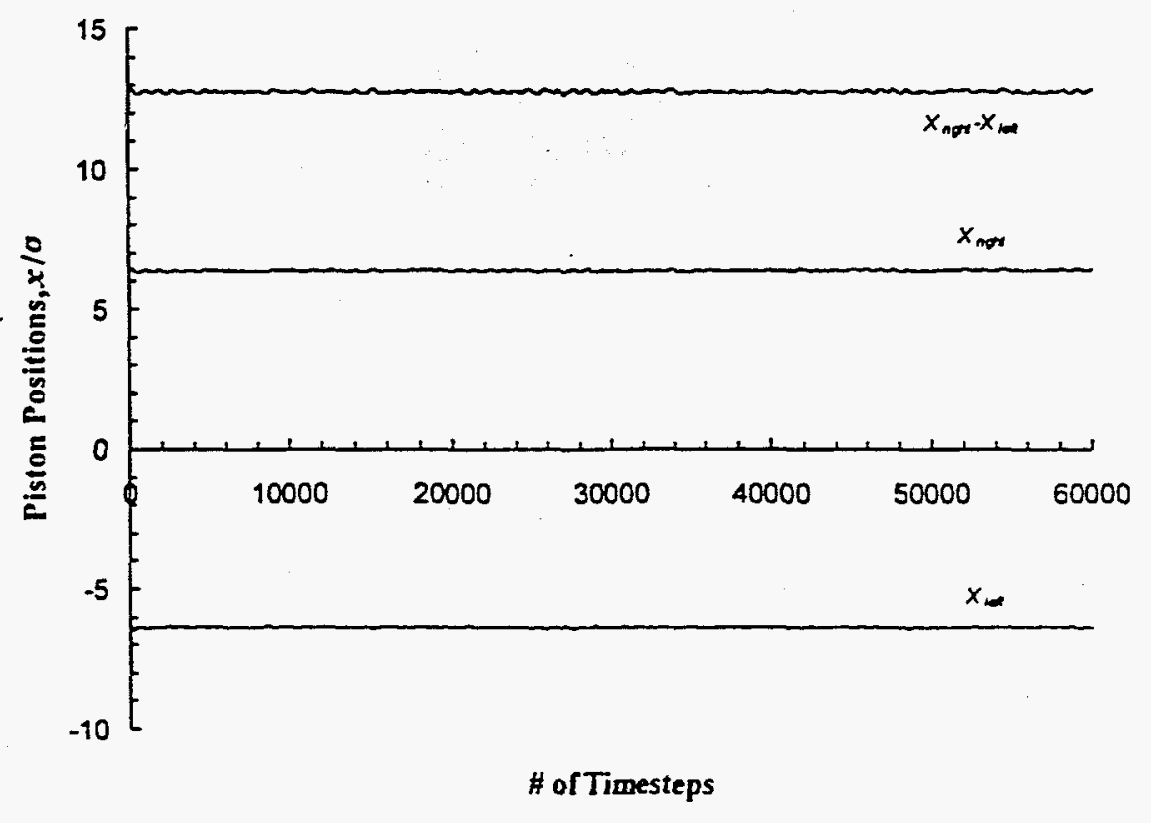

Figure 3. Short-time piston dynamics. Plot of the right piston position versus time for the first 50 timesteps, for $m_{w} / m_{f}$ ratios of 1 (diamonds), 10 (squares), and 100 (triangles). The fourth curve (circles) used a mass ratio of 1 , but with the $\mathrm{MD}$ timestep reduced by a factor of 10 , and only every tenth point plotted. All four simulations have the same starting point as the simulation shown in Fig. 2.

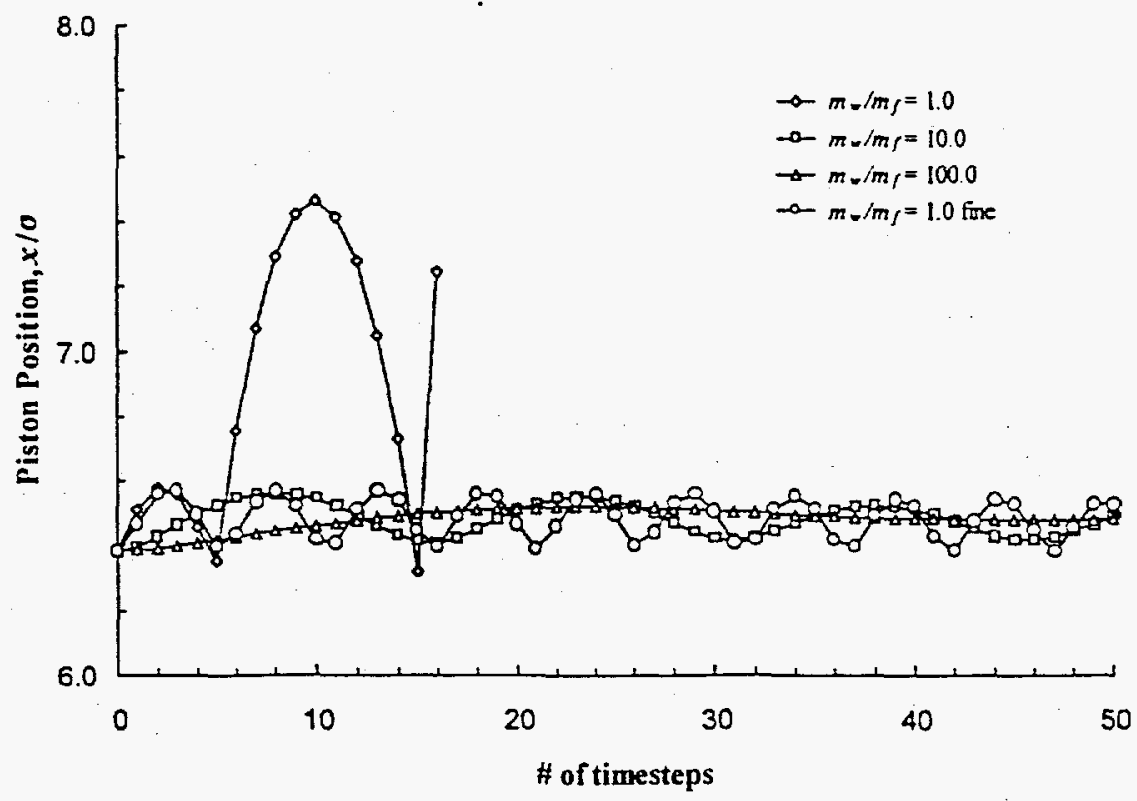


Figure 4. Long-time piston dynamics. Plot of right piston position versus time at 100 timestep intervals for $m_{\psi} / m_{f}$ ratios of 10 (squares), and 100 (triangles). The second curve is shifted upwards by $0.2 \sigma$ for clarity. Both simulations have the same starting point as the simulation shown in Fig. 2.

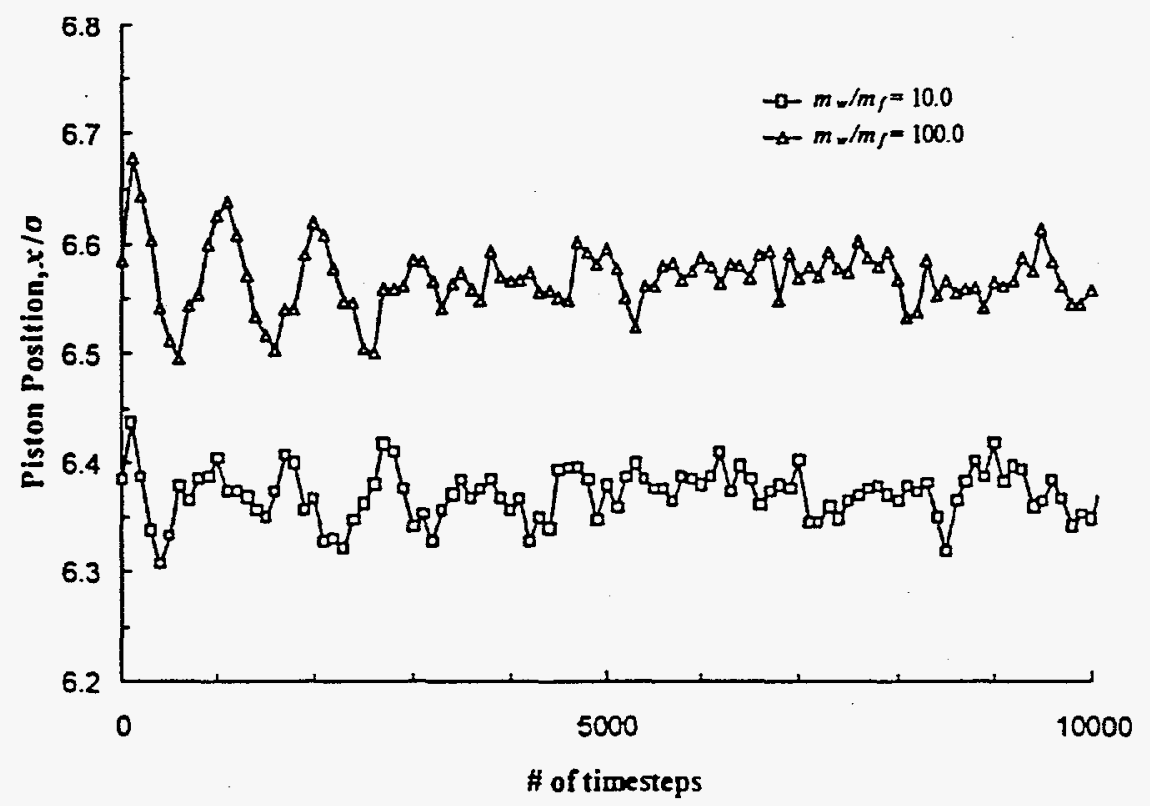

Figure 5. Time evolution of the concentration profile for component 2 in the constant-pressure DCV-GCMD simulation. Each line represents the average concentration profile accumulated over 20,000 timesteps; plot labels indicate the last timestep sampled. For clarity all the lines except the lowest have been offset vertically by successive increments of $\rho_{2} \sigma^{3}=0.1$. The grey lines represent the boundaries of the control volumes.

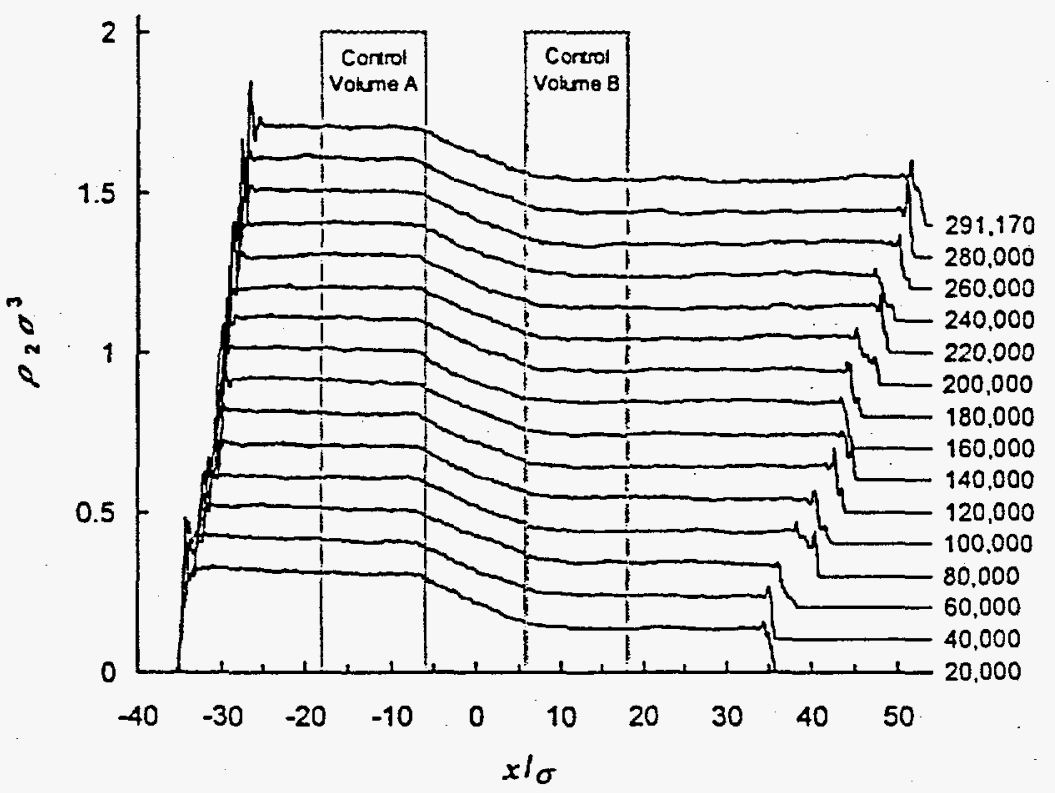


Figure 6. Average flux profiles sampled between timesteps 100,000 and 200,000 of the constantpressure DCV-GCMD simulation. The open squares are for component 1 and the filled squares are for component 2 . The fluxes are measured relative to the "stationary coordinate reference frame", i.e. the simulation box coordinates. The solid lines are the predictions of the continuum model described in the text. The grey lines represent the boundaries of the control volumes.

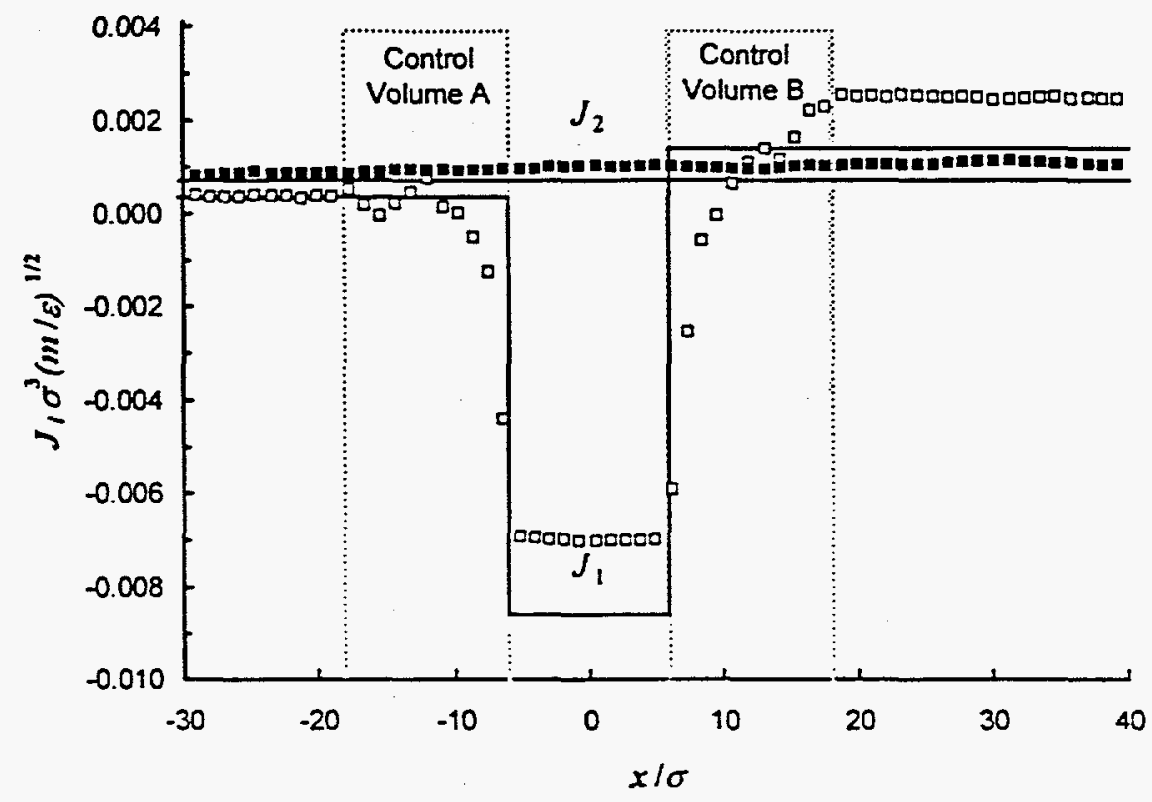

Figure 7. Plot of concentration gradient profiles (circles, left axis) and diffusion flux profiles (triangles, right axis) in the gradient zone from the constant-pressure color diffusion simulation. The open symbols are for component 1 and the filled symbols are for component 2 .

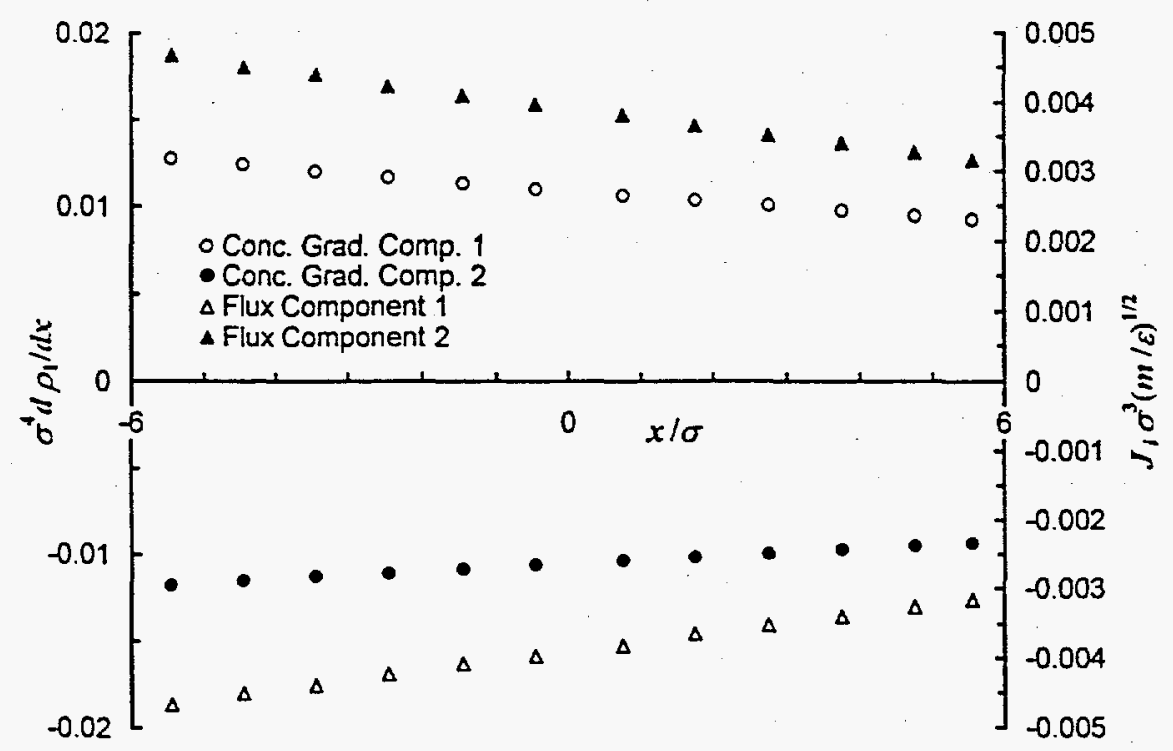


Figure 8. Diffusion coefficient profiles for components 1 (open circles) and 2 (filled circles) from the constant-pressure color diffusion simulation.

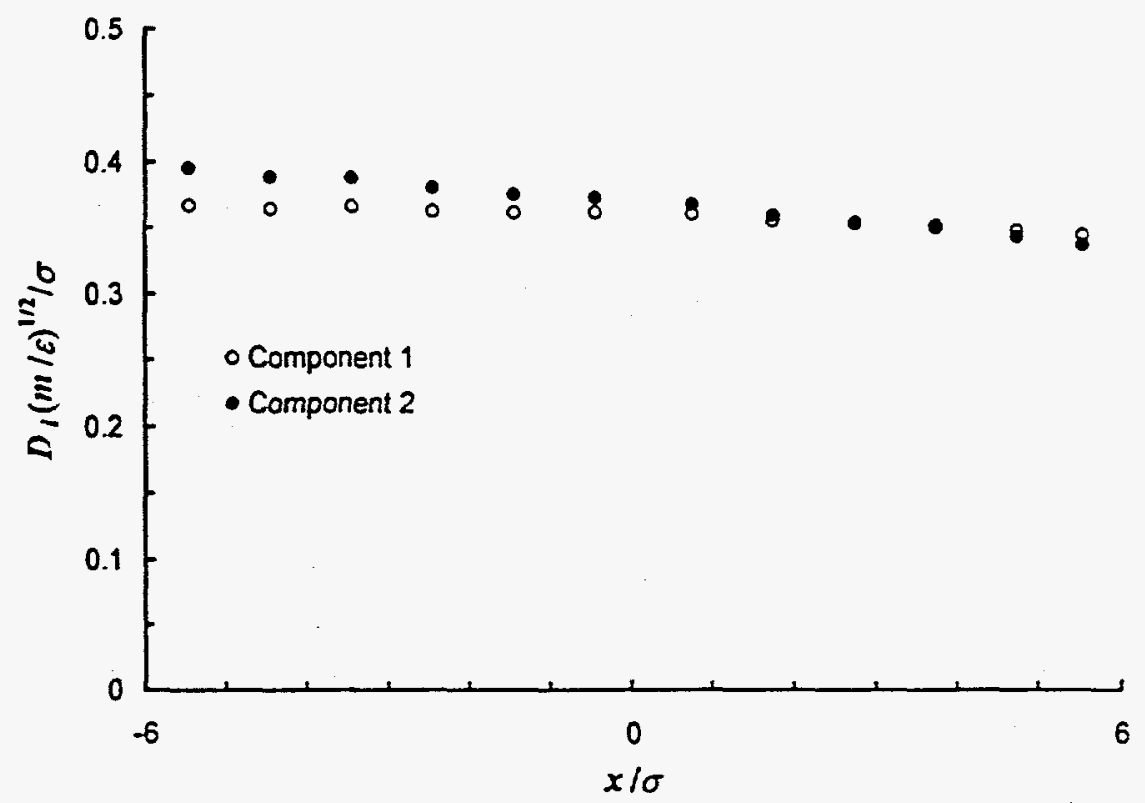

Figure 9. Concentration profiles from simulations of gradient-driven diffusion in a binary mixture of WCA spheres with a 1:2 size ratio. The open symbols are for component 1 (left axis) and the filled symbols are for component 2 (right axis). The circles are from the constant volume simulation, and squares are from the constant pressure simulation. The grey lines represent the boundaries of the control volumes.

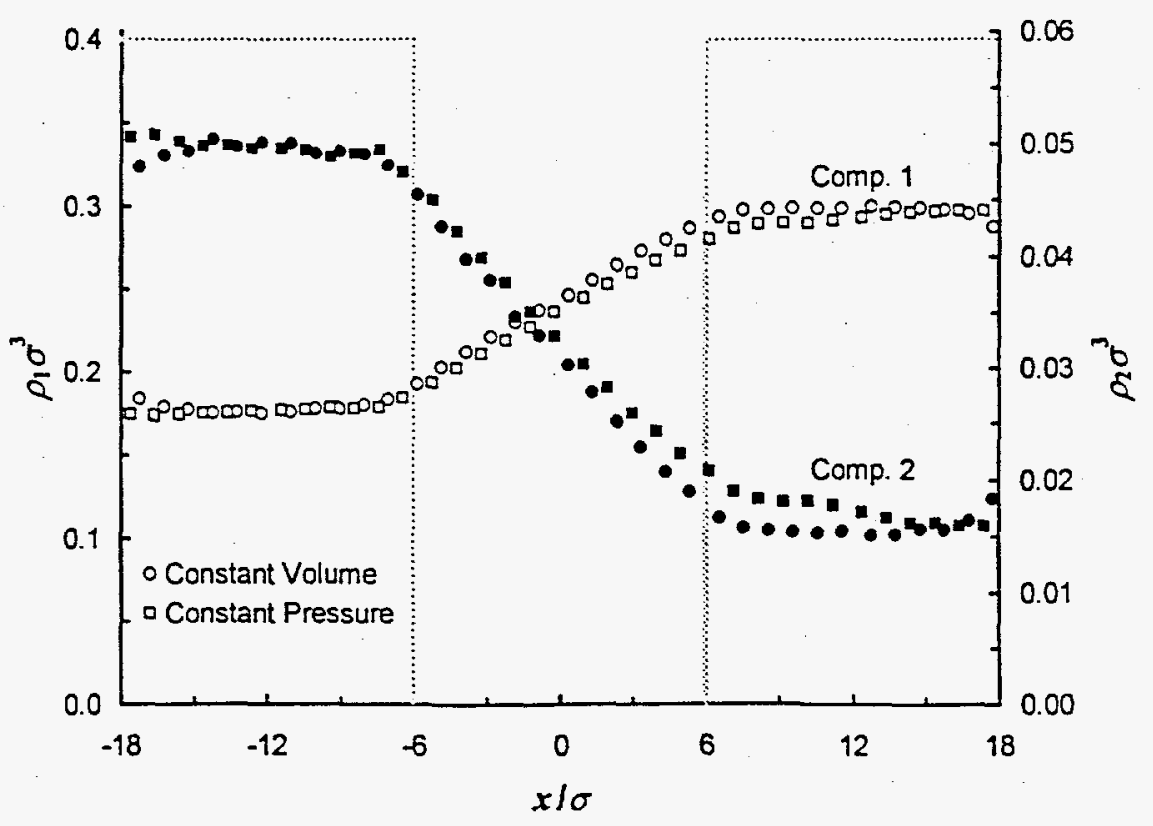


Figure 10. Flux profiles from simulations of gradient-driven diffusion in a binary mixture of WCA spheres with a 1:2 size ratio. The open symbols are for component 1 (left axis) and the filled symbols are for component 2 (right axis). The circles are from the constant volume simulation, and squares are from the constant pressure simulation. The grey lines represent the boundaries of the control volumes.

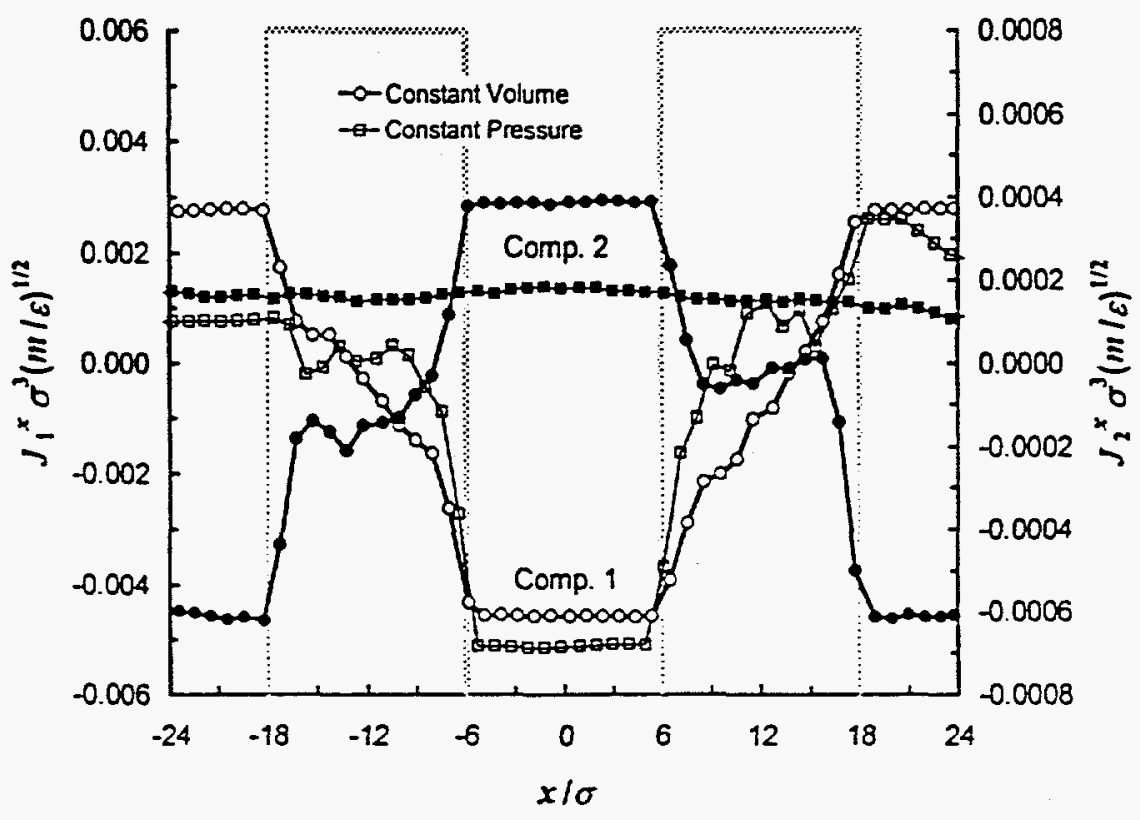

Figure 11. Binary diffusion coefficient profiles for the binary system of WCA spheres with a 1:2 size ratio. The open symbols are for component $l$ and the filled symbols are for component 2 . The circles are from the constant volume simulation (central gradient zone), and squares are from the constant pressure simulation.

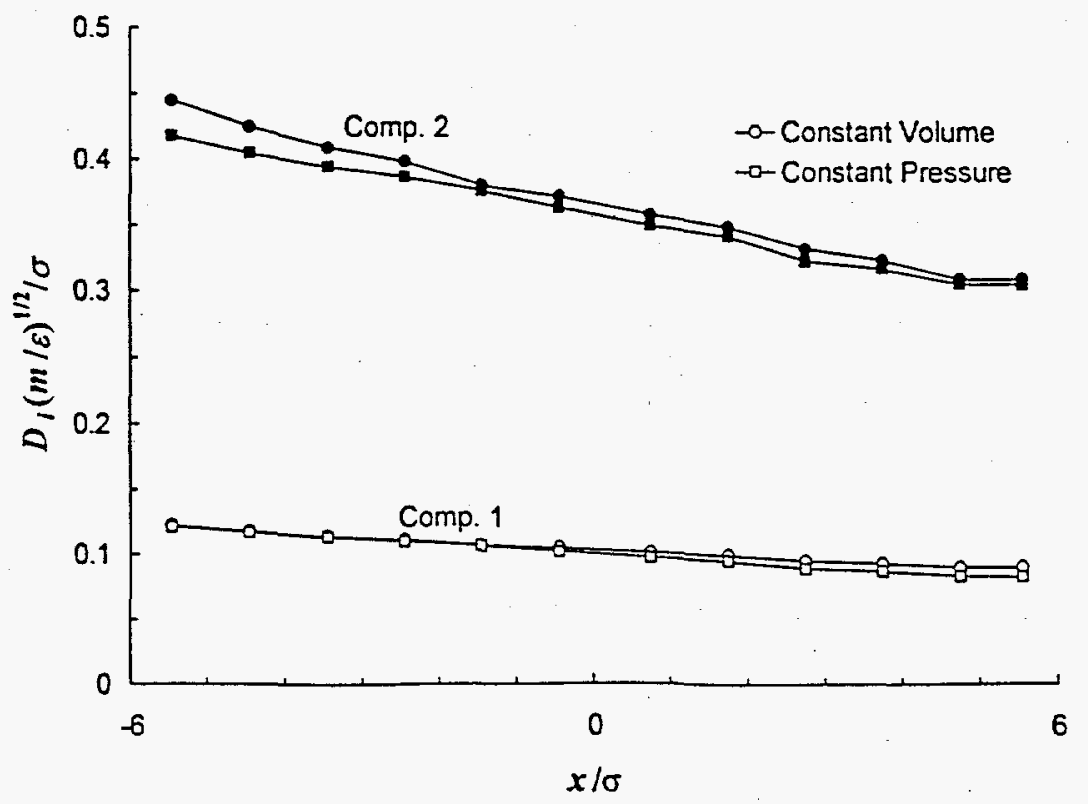


Figure 12. Chemical potential profiles for the binary system of WCA spheres with a 1:2 size ratio. The open symbols are for component 1 (left axis) and the filled symbols are for component 2 (right axis). The circles are from the constant volume simulation (central gradient zone), and squares are from the constant pressure simulation (component 1 only).

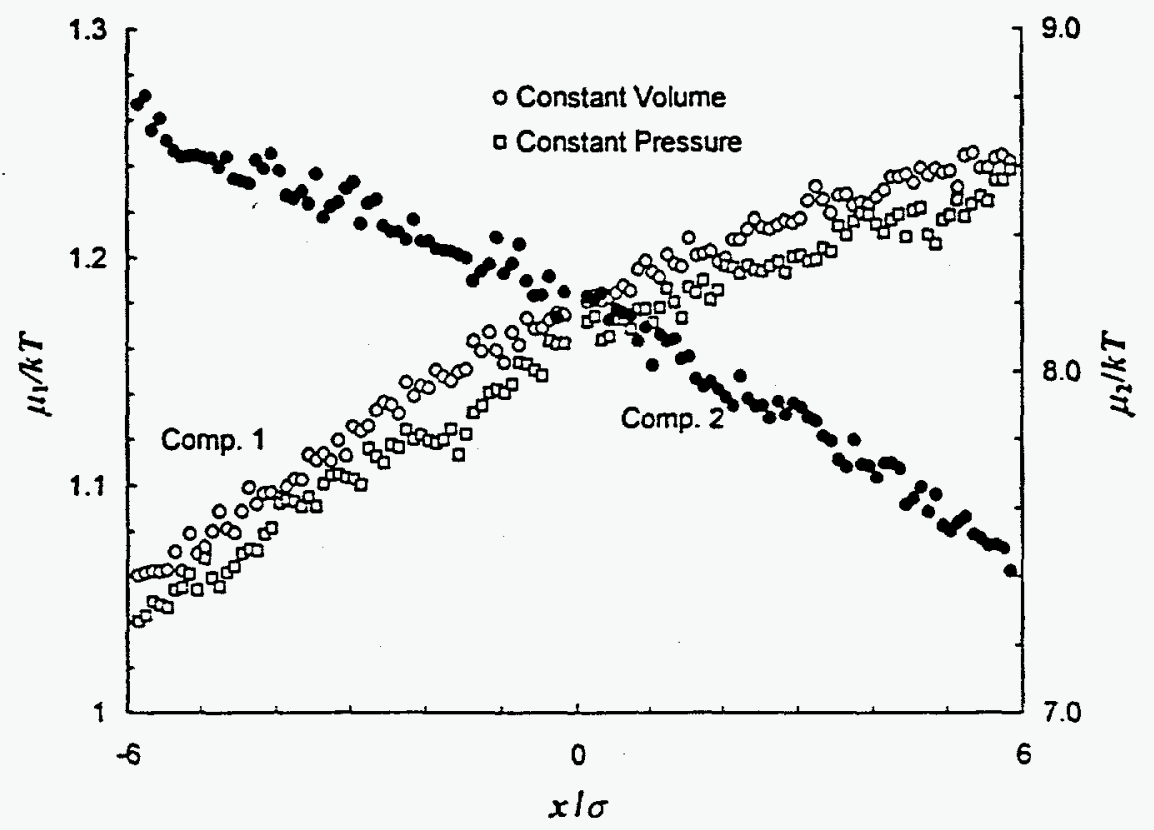

Figure 13. Profiles of the thermodynamic driving forces from the constant volume simulation of the binary system of WCA spheres with a 1:2 size ratio. The open circles are for component 1 and the closed circles are for component 2 . The triangles are the sum of the two forces. The solid lines are the profiles in the central gradient zone; the dashed lines are the profiles from the periodic gradient zone (with the $x$ coordinate shifted and reversed appropriately). The error bars are based on standard error estimates from the piecewise linear fits used to calculate the local chemical potential gradients.

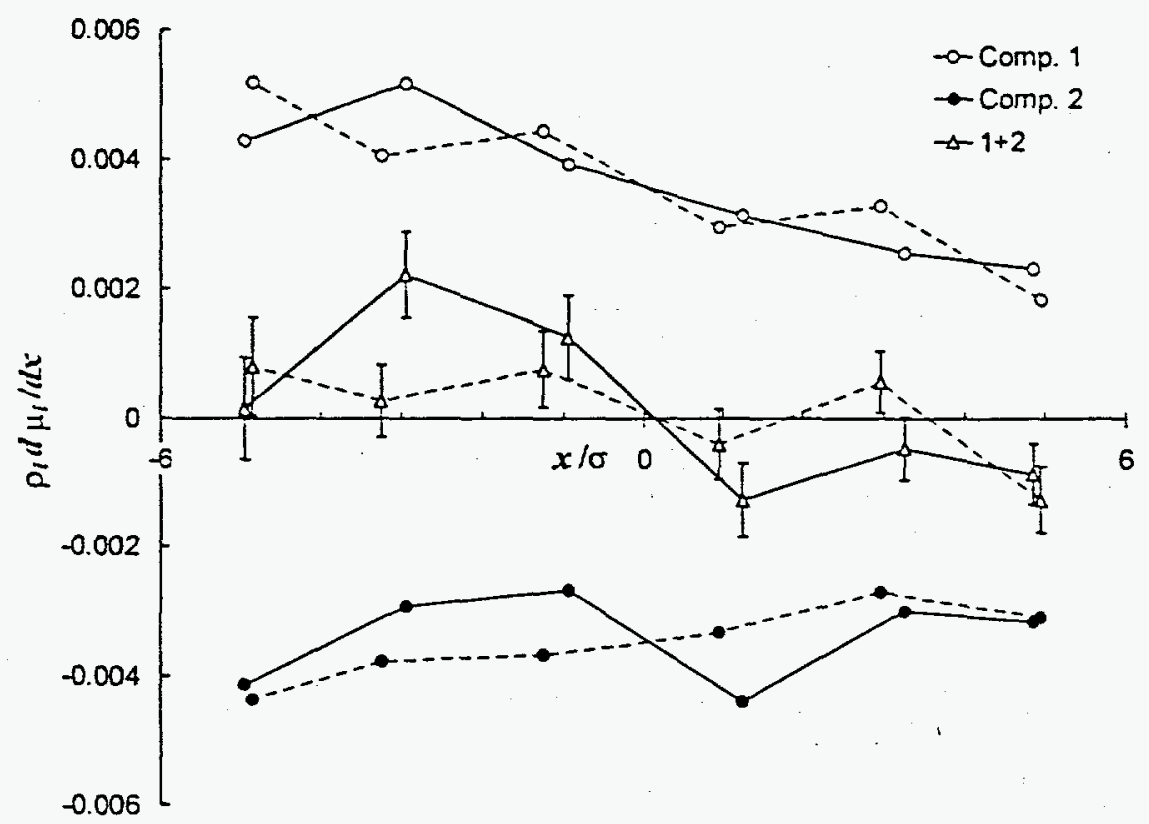


Figure 14. Maxwell-Stefan diffusion coefficient profiles for component 1 in the binary system of WCA spheres with a 1:2 size ratio. The circles are from the constant-volume simulation, with the solid line indicating the central gradient zone and the dashed line indicating the periodic gradient zone (with the $*$ coordinate shifted and reversed appropriately). The squares are from the constant-pressure simulation. The error bars are based on standard error estimates from the piecewise linear fits used to calculate the local chemical potential gradients.

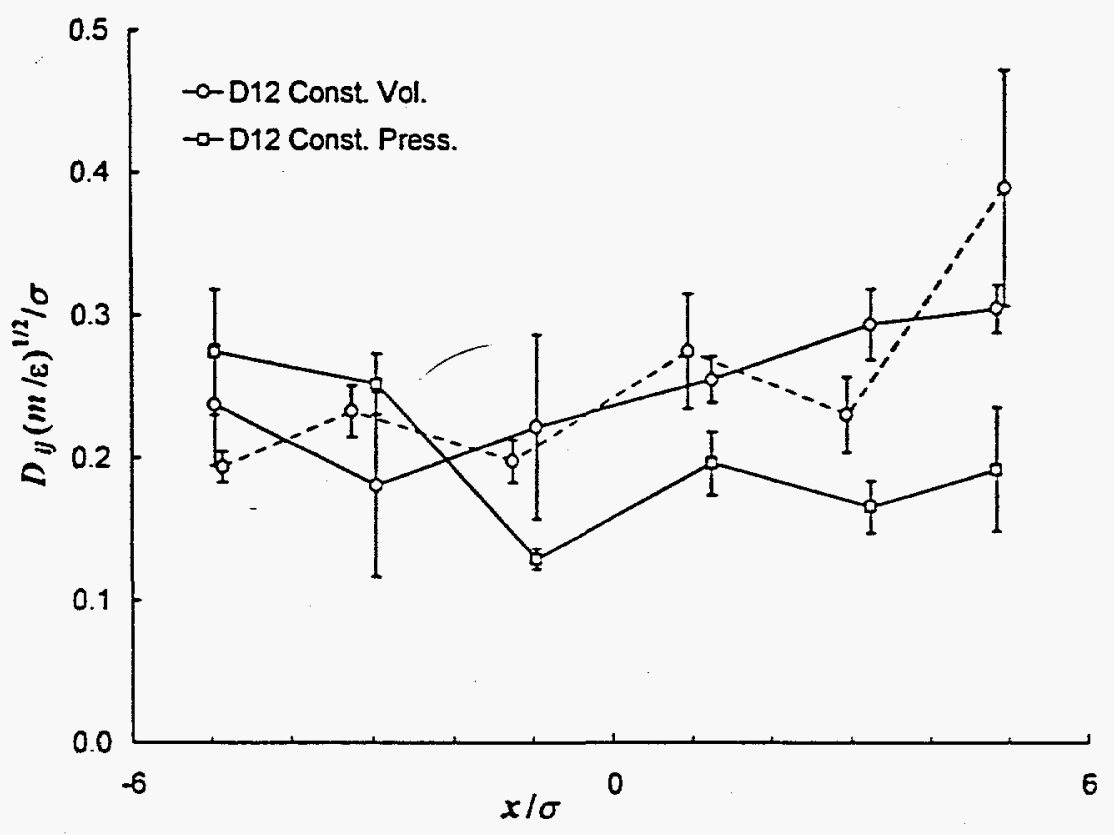

Figure 15. Maxwell-Stefan diffusion coefficient profiles for component 2 in the binary system of WCA spheres with a 1:2 size ratio. All data are from the constant-volume simulation, with the solid line indicating the central gradient zone and the dashed line indicating the periodic gradient zone (with the $x$ coordinate shifted and reversed appropriately). The error bars are based on standard error estimates from the piecewise linear fits used to calculate the local chemical potential gradients.

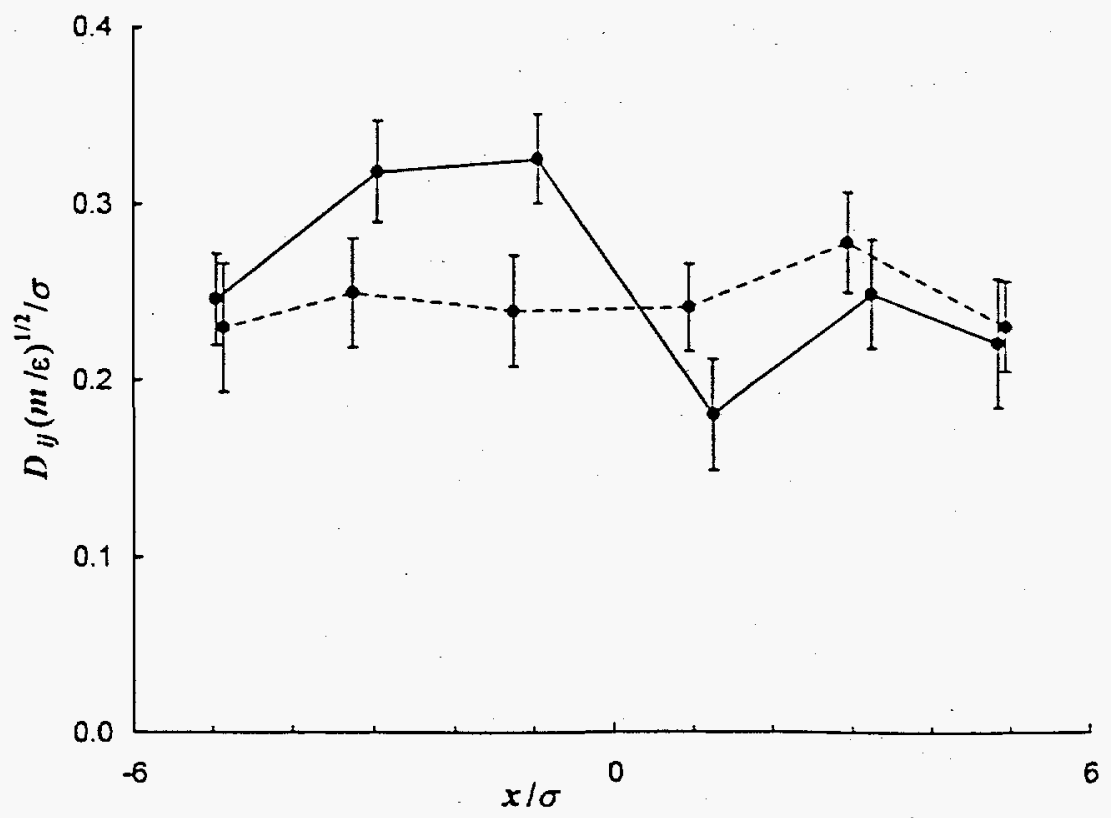

